\title{
Myosin X Interaction with KIF13B, a Crucial Pathway for Netrin-1-Induced Axonal Development
}

\author{
Hua-Li Yu, ${ }^{1,2,3 *}$ Yun Peng, ${ }^{2 *}$ Yang Zhao, ${ }^{1,3}$ Yong-Sheng Lan, ${ }^{1,2}$ Bo Wang, ${ }^{1,2}$ Lu Zhao, ${ }^{1,2,3}$ Dong Sun, ${ }^{1}$ Jin-Xiu Pan, ${ }^{1}$ \\ Zhao-Qi Dong, ${ }^{1}$ Lin Mei, ${ }^{1,2}$ Yu-Qiang Ding, ${ }^{4}{ }^{\circledR}$ Xiao-Juan Zhu, ${ }^{3}$ and ${ }^{\circledR}$ Wen-Cheng Xiong ${ }^{1,2}$ \\ ${ }^{1}$ Department of Neurosciences, School of Medicine, Case Western Reserve University, Cleveland, Ohio 44106, ${ }^{2}$ Department of Neuroscience and \\ Regenerative Medicine, Medical College of Georgia, Augusta University, Augusta, Georgia 30912, ${ }^{3}$ Institute of Cytology and Genetics, Northeast \\ Normal University, Changchun 130024, People's Republic of China, and ${ }^{4}$ Institute of Brain Sciences, Fudan University, Shanghai 200031, People's \\ Republic of China
}

Myosin X (Myo X) transports cargos to the tips of filopodia for cell adhesion, migration, and neuronal axon guidance. Deleted in Colorectal Cancer (DCC) is one of the Myo X cargos that is essential for Netrin-1-regulated axon pathfinding. The function of Myo $\mathrm{X}$ in axon development in vivo and the underlying mechanisms remain elusive. Here, we provide evidence for the role of Myo X in Netrin-1-DCC-regulated axon development in developing mouse neocortex. The knockout (KO) or knockdown (KD) of Myo X in cortical neurons of embryonic mouse brain impairs axon initiation and contralateral branching/targeting. Similar axon deficits are detected in Netrin-1-KO or DCC-KD cortical neurons. Further proteomic analysis of Myo X binding proteins identifies KIF13B (a kinesin family motor protein). The Myo X interaction with KIF13B is induced by Netrin-1. Netrin-1 promotes anterograde transportation of Myo X into axons in a KIF13B-dependent manner. KIF13B-KD cortical neurons exhibit similar axon deficits. Together, these results reveal Myo X-KIF13B as a critical pathway for Netrin-1promoted axon initiation and branching/targeting.

Key words: axon; KIF13B; Myo X; Netrin-1; KIF13B; axon initiation; axon branching

Significance Statement

Netrin-1 increases Myosin X (Myo X) interaction with KIF13B, and thus promotes axonal delivery of Myo X and axon initiation and contralateral branching in developing cerebral neurons, revealing unrecognized functions and mechanisms underlying Netrin-1 regulation of axon development.

\section{Introduction}

Neurons are highly polarized cells typically with a single axon and multiple dendrites. Axon development is crucial for the establishment of neuronal connections, especially for the connection between different brain regions. Axon development includes the following three main steps: (1) axon specification/initiation during neuronal polarization; (2) axon growth and guidance;

Received Apr. 23, 2020; revised Sep. 4, 2020; accepted 0ct. 17, 2020.

Author contributions: L.M. and W.-C.X. designed research; H.L.-Y., Y.P., Y.Z., Y.-S.L., B.W., L.Z., D.S., J.-X.P., and Z.-Q.D. performed research; Y.-Q.D. and X.-J.Z. contributed unpublished reagents/analytic tools; H.L.-Y. and Y.P. analyzed data; H.L.-Y. wrote the paper.

${ }^{*}$ H.L.-Y. and Y.P. have contributed equally to this work.

This project is supported in part by the National Institute on Aging/National Institutes of Health (Grant AG045781). We thank Dr. Hiroaki Miki (Osaka University, Osaka, Japan) for providing KIF13B reagents.

The authors declare no competing financial interests.

Correspondence should be addressed to Wen-Cheng Xiong at Wen-Cheng.Xiong@case.edu or Xiao-Juan Zhu at zhuxj720@nenu.edu.cn.

https://doi.org/10.1523/JNEUROSCI.0929-20.2020

Copyright (c) 2020 Yu et al.

This is an open-access article distributed under the terms of the Creative Commons Attribution 4.0 International license, which permits unrestricted use, distribution and reproduction in any medium provided that the original work is properly attributed. and (3) axon branching and presynaptic differentiation. During early development of mammalian cortex, the migrating neurons in the intermediate zone (IZ) transit from multipolar to bipolar, with a leading process toward the pia and a trailing process toward the ventricle. Once localized into the cortical plate (CP), the leading processes will develop into highly branched dendrites and the trailing processes will become long axons projecting to target regions for further bifurcation (Barnes and Polleux, 2009; Yogev and Shen, 2017). Eventually, axons connect with target neurons to form synapses that are crucial for neurotransmission.

Axon development is regulated by intrinsic factors in neurons as well as by microenvironmental factors or extracellular guidance cues. Netrin-1 belongs to the netrin family of extracellular guidance cues, which is crucial for axon pathfinding (Colamarino and Tessier-Lavigne, 1995; Braisted et al., 2000). Netrin-1 exerts attractive and repulsive effects through two families of receptors, Deleted in Colorectal Cancer (DCC) and UNC-5, respectively (Hedgecock et al., 1990; Ackerman et al., 1997; Leonardo et al., 1997; Culotti and Merz, 1998; Hong et al., 1999; Keleman and Dickson, 2001). Various signaling cascades are involved in Netrin1-DCC-induced neurite outgrowth and/or growth cone guidance in 
cultured neurons, which include Rho family GTPases (Li et al., 2002; Shekarabi and Kennedy, 2002), phospholipase C (Xie et al., 2006), phosphoinositol 3-kinase (PI3K; Ming et al., 1999), extracellular signal-regulated protein kinase (Campbell and Holt, 2003; Forcet et al., 2002; Ming et al., 2002), FAK (Li et al., 2004; Liu et al., 2004; Ren et al., 2004), PITPalpha (Xie et al., 2005), and Myosin X (Myo X; Zhu et al., 2007). Recent studies using Netrin-1 CKO mice suggest that the ventricular zone-derived Netrin-1 contributes to commissural axon projection by bounding to commissural axons near the pial surface (Dominici et al., 2017). This finding makes the function of locally produced Netrin-1 in commissural axon development more prominent. However, how Netrin-1 regulates cortical neuronal axon development or projection remains to be determined.

Myo X, an unconventional actin-based motor protein, is primarily localized at the tips of filopodia or the edges of lamellipodia and membrane ruffles (Berg et al., 2000; Berg and Cheney, 2002; Tokuo and Ikebe, 2004; Zhang et al., 2004). It undergoes forward and rearward movements within filopodia and promotes filopodia formation, elongation, and sensing, possibly by transporting actin binding proteins and cell adhesion receptors to the leading edge of the cells (Berg and Cheney, 2002; Tokuo and Ikebe, 2004; Zhang et al., 2004; Tokuo et al., 2007; Zhu et al., 2007). Myo X has a unique protein structure feature, containing a motor domain at its $\mathrm{N}$ terminus, three calmodulin-binding IQ motifs, $3 \mathrm{PH}$ (pleckstrin homology) domains, one myosin tail homology (MyTH) domain, and one band 4.1-ezrin-radixinmeosin (FERM) domain (Berg et al., 2000; Yonezawa et al., 2000). Via these domains, Myo X not only binds to F-actin filaments, but also interacts with phosphoinositol lipids, microtubules, and transmembrane receptors (e.g., integrins and DCC family receptors; Cox et al., 2002a; Tokuo and Ikebe, 2004; Weber et al., 2004; Zhang et al., 2004; Zhu et al., 2007; Plantard et al., 2010). In cultured neurons, Myo X is gradually accumulated in nascent axons, where it regulates axon outgrowth (Yu et al., 2012). In chicken embryos, expression of motorless Myo X impairs axon growth and commissural axon midline crossing (Zhu et al., 2007). Notice that Myo X is critical for transporting DCC to the dynamic actin-based membrane protrusions (Zhu et al., 2007), and, on the other hand, the motor activity and distribution of Myo X are also reciprocally regulated by DCC and neogenin (Liu et al., 2012). Whereas these observations support the view for Netrin-1-DCC-Myo X pathway to be critical for axon development, whether and how they regulate axon development in vivo remain to be further elucidated.

Here, we present evidence for Myo X interaction with KIF13B to be crucial for Netrin-1-induced axon initiation and branching/ targeting in developing mouse cortical brain. Myo X interacts with KIF13B (also called GAKIN), a kinesin family member that is essential for delivery of phosphatidylinositol $(3,4,5)$-trisphosphate [PI $(3,4,5) \mathrm{P} 3]$ to axons and axon outgrowth (Horiguchi et al., 2006; Yoshimura et al., 2010). Netrin-1 increases Myo X interaction with KIF13B, thus promoting anterograde transport and axonal targeting of Myo X in neurons. Such Netrin-1 function requires Myo X interaction with DCC and KIF13B, as well as PI3K activity. Additionally, as Netrin-1 and DCC, both Myo X and KIF13B are required for axon initiation and contralateral branching/targeting in developing cerebral cortex. Together, these results suggest that by promoting KIF13B-mediated axonal transport of Myo X, Netrin-1 plays critical roles in inducing axonal initiation and enhancing axonal contralateral branching, revealing unrecognized functions and mechanisms underlying the Netrin-1 signaling pathway in axon development.

\section{Materials and Methods}

Animals. Myo $\mathrm{X}^{\mathrm{f} / \mathrm{f}}$ mice were generated as previously described (Wang et al., 2019), and Netrin-1 floxed (NTN1 $1^{\mathrm{f} / \mathrm{f}}$ ) mice were kindly provided by Yu-Qiang Ding (Fudan University, Shanghai, China). All the mouse lines indicated above were maintained on a C57BL/6 background for more than six generations. Timed pregnant female mice were obtained by crossing with male mice overnight, and the noon of the following day was designated as embryonic day 0.5 (E0.5) if the vaginal plug was detected.

Reagents. For immunostaining analysis, the following primary antibodies were used: mouse monoclonal anti-Tau-1 (catalog \#05-838; 1:1000), mouse monoclonal anti-MAP2 (catalog \#05-346; 1:500), rat monoclonal anti-tubulin (catalog \#MAB1864; 1:500) from Millipore; chicken polyclonal anti-GFP (catalog \#ab13970; 1:1000) and mouse monoclonal anti-c-Myc (catalog \#ab32; 1:200) from Abcam. For immunoblotting analysis, the following primary antibodies were used: goat polyclonal anti-DCC (catalog \#sc-6535; 1:200) from Santa Cruz Biotechnology; rabbit polyclonal anti-KIF13B (catalog \#PA540807;1:500) from Thermo Fisher Scientific; mouse monoclonal anti- $\alpha$-tubulin (catalog \#T5168; 1:4000) and mouse monoclonal anti-GFP (catalog \#11814460001; 1:2000) from Sigma-Aldrich; rabbit polyclonal anti-Myc (catalog \#ab9106; 1:1000) from Abcam; rabbit polyclonal anti-MyoX was prepared as previously described (Zhu et al., 2007); the polyclonal anti-KIF13B antibody was provided by Hiroaki Miki (Osaka University, Osaka, Japan). For immunoprecipitation assay, rabbit polyclonal anti-GFP (catalog \#A11122) was purchased from Thermo Fisher Scientific. Alexa Fluor 488-, 555-, and 647coupled secondary antibodies against mouse, rat, chicken, or goat and HRP-conjugated secondary antibodies against mouse or rabbit were purchased from Jackson ImmunoResearch. Alexa Fluor 350-phalloidin (catalog \#A22281) and lipofectamine 2000 (catalog \#11668-019) were obtained from Thermo Fisher Scientific. Wortmannin was from Millipore.

Expression vectors. The cDNA of mouse Myo $X$ was subcloned into mammalian expression vector (pEGFP-C1) fused with GFP at the $\mathrm{N}$ terminus, as described previously (Zhu et al., 2007). GFP-MyoX $\Delta \mathrm{PH}$ and GFP-MyoX $\triangle$ FERM were generated with a Q5 Site-Directed Mutagenesis Kit (catalog \#E0554S, New England Biolabs), where the amino acids 1212-1253 and 1799-2058 were deleted, respectively. Myo X-Head, Myo X $\Delta$ Motor (headless Myo X, hMyo X), Myo X MyTH4FERM, and MyoX FERM were amplified and subcloned into pEGFPC1. GFP-hMyoX $\triangle \mathrm{PH} 2$ and GFP-hMyoX $\Delta \mathrm{PH} 3$ were generated with the Q5 Site-Directed Mutagenesis Kit, where the amino acids 1215-1316 and 1381-1506, respectively, were deleted. Also, GFP-hMyoX (KK1225/ 6AA) were generated with the Q5 Site-Directed Mutagenesis Kit. The cDNA of human KIF13B was subcloned into mammalian expression vector (pRK5) fused with a Myc tag at the $\mathrm{N}$ terminus. KIF13B ${ }^{1-557}$, KIF13B ${ }^{1-1531}$, KIF13B ${ }^{558-1826}$, KIF13B ${ }^{990-1826}$, and KIF13B ${ }^{1532-1826}$ were amplified by PCR and subcloned into pRK5 through corresponding restriction enzymes or exonuclease III. The expression vectors of all glutathione $S$-transferase (GST) fusion proteins was constructed by ligation into pGEX-6p-1. mCherry-Myo X was kindly provided by Staffan Strömblad (Karolinska Institutet, Huddinge, Sweden).

The miRNA expression vectors for Myo X and DCC were generated by the BLOCK-iT Lentiviral miR RNA Expression System (Thermo Fisher Scientific) according to the manufacturer instruction as previously described (Zhu et al., 2007; Liu et al., 2012). The shRNA expression vectors for mouse KIF13B were generated using pll3.7 lentiviral vector, and the target sequences for KIF13B shRNAs are as follows: $5^{\prime}$-GCA GATAACTATGACGAAACC-3' (KIF13B shRNA-1); and 5'-GGATTT AGCTGGCAGTGAACG-3' (KIF13B shRNA-2). Netrin-1 shRNA was constructed into pll3.7 lentiviral vector as previously reported, and the target sequence was 5'-GGGTGCCCTTCCAGTTCTA-3' (Chen et al., 2017). DCC shRNA was constructed into pll3.7 lentiviral vector as previously reported, and the target sequence was 5 -CTTGGAGGAAGGAG AGACA-3' (Zhang et al., 2018). In addition, we also generated the RFP-Scramble shRNA and RFP-KIF13B shRNA expression vectors by replacing GFP with RFP in the pll3.7 plasmids. The authenticity of all constructs was verified by DNA sequence.

Cell cultures and transfections. Primary cortical neurons were cultured as described previously (Zhu et al., 2007). In brief, embryos (E17) were removed from anesthetized pregnant mice. Cerebral cortices were 
separated and chopped into small pieces. After incubation in $0.125 \%$ Trypsin plus with $0.05 \%$ DNase in HBSS at $37^{\circ} \mathrm{C}$ for $20 \mathrm{~min}$, cells were triturated with a fire-polished glass Pasteur pipette and filtered with a $40 \mu \mathrm{m}$ filter. Dissociated cells were suspended in DMEM with 10\% FBS and plated on poly-D-lysine-coated dishes or glass coverslips at $37^{\circ} \mathrm{C}$ in a $5 \% \mathrm{CO}_{2}$ atmosphere. Four hours later, the medium was changed into Neurobasal medium with 2\% B27 supplement and 2 mм Glutamax. For transfection, neurons were electroporated immediately after dissociation using the Mouse Neuron Nucleofector Kit (Amaxa). In brief, $3 \times 10^{6}$ neurons were resuspended in $100 \mu \mathrm{l}$ of Nucleofectamine solution containing $3 \mu \mathrm{g}$ of plasmid and electroporated with Program O-003 of Nucleofector.

NLT cells and HEK293 cells were grown in DMEM supplemented with $10 \% \mathrm{FBS}$ and $100 \mathrm{U} / \mathrm{ml}$ penicillin-streptomycin. For imaging experiments, $50-70 \%$ confluent NLT cells in 12 -well plate were transfected with $1.6 \mu \mathrm{g}$ of indicated plasmids using $3 \mu \mathrm{l}$ of lipofectamine in DMEM without FBS and antibiotics. For Western blot and coimmunoprecipitation, HEK293 cells were transfected using polyetherimide (PEI). Stable HEK293 cell line expressing human Netrin-1 was used as described previously (Ren et al., 2004; Xie et al., 2005; Zhu et al., 2007). The working concentration of Netrin-1 was $500 \mathrm{ng} / \mathrm{ml}$.

In utero electroporation. The in utero electroporation (IUE) was conducted as described previously with some modifications ( $\mathrm{Yu}$ et al., 2012). Briefly, plasmids were microinjected into the lateral cerebral ventricle of E14.5 or E15.5 mouse embryos through the uterine wall. Then, a $35 \mathrm{~V}$ square-wave pulse was delivered across the head for five times through and ECM-830 Electroporation System (BTX). Embryos were then allowed to develop to E18.5, postnatal day 7 (P7) or P14. The transfected brains were then fixed with $4 \% \mathrm{PFA} / \mathrm{PBS}$ overnight at $4^{\circ} \mathrm{C}$. The brains were sectioned with a freezing microtome at $\sim 50 \mu \mathrm{m}$.

Immunostaining analysis. Cells were fixed in $4 \%$ PFA for $10 \mathrm{~min}$ at room temperature, permeabilized with $0.1 \%$ Triton X-100 for $8 \mathrm{~min}$, and blocked in $2 \%$ bovine serum albumin for $1 \mathrm{~h}$ in $0.01 \mathrm{M}$ PBS, $\mathrm{pH}$ 7.4. Subsequently, cells were incubated with primary antibodies diluted in the blocking solution for $2 \mathrm{~h}$ and washed three times with PBS. And they were incubated with appropriate fluorochrome-conjugated secondary antibodies for $1 \mathrm{~h}$ and washed three times.

Live cell time-lapse imaging and kymography analyses. Transfected neurons were grown on Lab-Tek II Chambered cover glass (Thermo Fisher Scientific) in DMEM supplemented with 10\% FBS and antibiotics. For visualizing GFP-Myo X movement, the Lab-Tek II Chambered Coverglass were then fitted into a temperature-controlled chamber on the microscope stage of LSM 710 confocal laser-scanning microscopy (Carl Zeiss) for observation at $37^{\circ} \mathrm{C}$ in a $5 \% \mathrm{CO}_{2}$ atmosphere. The timelapse intervals were $5 \mathrm{~s}$, and neurons were imaged over periods of $10 \mathrm{~min}$. Imagines were acquired with a $63 \times / 1.4$ numerical aperture (NA) objective at a resolution of $1024 \times 1024$ pixels. The software Image (FIJI) was used for tracking analysis and kymographic analysis. In brief, the traveling path and velocity of GFP-Myo X puncta were recorded with the "Manual Tracking" plugin by clicking on the GFPMyo X puncta on the temporal stacks. For kymographic analysis, a segmented line was used to draw a region of interest (ROI) and then the "KymographBuilder" plugin was used to produce kymographs for the selected segments.

Fluorescence recovery after photobleaching. The experiments were performed using the LSM 710 confocal laser-scanning microscopy. Images were acquired with an $63 \times / 1.4$ N.A. objective at a resolution of $1024 \times 1024$ pixels. A region at the proximal axon was bleached with high laser power, and fluorescence recovery was observed for a period of $10 \mathrm{~min}$. For fluorescence recovery after photobleaching (FRAP) analysis, the mean intensity of the bleached area was normalized with the initial fluorescence intensity before bleaching.

Mass spectrum analysis. Cultured cortical neurons transfected with GFP-hMyoX expression plasmids were lysed $48 \mathrm{~h}$ later with lysis buffer [20 mм Tris, pH 8.0, 10 mм NaCl, 1 mм EDTA, 0.5\% NP-40, 1 mм NaF, $1 \mathrm{mM} \mathrm{Na}_{3} \mathrm{VO}_{4}$, protease inhibitor cocktail (Roche), $1 \mathrm{~mm}$ PMSF]. GFP$\mathrm{hMyoX}$ and its binding partners were immunoprecipitated with antiIgG (control) or anti-GFP antibodies, respectively. The immunoprecipitates were resolved by $8 \%$ SDS-PAGE gel and subjected to silver staining.
All unique bands in anti-GFP group at molecular weight from $\sim 50-250$ $\mathrm{kDa}$ on the gel were isolated and subjected to liquid chromatographytandem mass spectrometry (LC-MS/MS) as previously reported (Ma et al., 2019; Applied Protein Technology). Briefly, gel pieces were destained, rehydrated, and tryptic digested with $10 \mathrm{ng} / \mu \mathrm{l}$ trypsin resuspended in 50 $\mathrm{mm} \mathrm{NH}_{4} \mathrm{HCO}_{3}$. The tryptic peptides were dissolved in $0.1 \%$ formic acid (solvent A), directly loaded onto a reversed-phase analytical column. The gradient was composed of an increase from $6 \%$ to $23 \%$ solvent B ( $0.1 \%$ formic acid in $98 \%$ acetonitrile). The peptides were subjected to nanospray ionization (NSI) source followed by MS/MS in Q Exactive Plus (Thermo Fisher Scientific) coupled online to the ultra-performance LC. The resulting MS/MS data were processed using Proteome Discoverer 1.3. Tandem mass spectra were searched against UniProt database. Trypsin was specified as a cleavage enzyme allowing up to two missing cleavages. Mass error was set to $10 \mathrm{ppm}$ for precursor ions and $0.02 \mathrm{Da}$ for fragment ions. Carbamidomethyl on Cys were specified as fixed modification and oxidation on Met and acetylation modification were specified as variable modifications. Peptide confidence was set at high, and peptide ion score was set $>20$.

Protein-protein interaction assays. Immunoprecipitation was conducted as previously described (Ren et al., 2001). Cell lysates (1 mg of protein) were incubated at $4^{\circ} \mathrm{C}$ for $6 \mathrm{~h}$ with the indicated antibodies $(1-2 \mu \mathrm{g})$ in a final volume of $1 \mathrm{ml}$ modified RIPA lysis buffer with protease inhibitors. After the addition of protein A-G-agarose beads, each reaction was incubated at $4^{\circ} \mathrm{C}$ for $1 \mathrm{~h}$. The immunoprecipitate complexes were collected by centrifugation and washed three times with washing buffer (20 mm Tris-HCl, $10 \mathrm{~mm} \mathrm{NaCl}, 1 \mathrm{~mm}$ EDTA, 0.5\% NP-40). Immune complexes were resolved by SDS-PAGE and subjected to immunoblotting. GST pulldown assay was conducted as described previously (Ren et al., 2001). Transiently transfected HEK293 cells were lysed in the modified RIPA buffer (50 mM Tris- $\mathrm{HCl}, \mathrm{pH}$ 7.4, $150 \mathrm{~mm}$ sodium chloride, $1 \% \mathrm{NP}-40,0.25 \%$ sodium-deoxycholate, and proteinase inhibitors). Cell lysates were precleared with GST immobilized on glutathione-Sepharose 4B (GE Healthcare) and then incubated with the indicated GST fusion proteins $(2-5 \mu \mathrm{g})$ immobilized on glutathioneSepharose beads at $4^{\circ} \mathrm{C}$ overnight with constant rocking. The beads were washed three times with modified RIPA buffer, and bound proteins were resolved by SDS-PAGE and subjected to immunoblotting.

Imaging quantification and statistical analyses. Immunostaining sections and cells were observed under a Zeiss LSM 710 confocal microscope with ZEN 2012 software, and only the brightness, contrast, and color balance were optimized after imaging. The software ImageJ was used to measure fluorescence intensity in all fixed images. The software ImageJ was used to measure fluorescence intensity in all fixed images. In brief, the RGB images were converted into 8-bit grayscale images and inverted to negative images for analysis. After being converted to uncalibrated optical density value, the area of axons, soma, and dendrites was selected with ROI tools and calculated. GFP-Myo X intensity in axons or dendrites was normalized by that in its soma regions. Statistical analyses were performed using either unpaired two-tailed Student's $t$ test or oneway ANOVA followed by a protected least significant difference Fisher's post hoc test for multiple comparisons. Statistical evaluations were performed with the software GraphPad Prism version 5.0. The data are presented as the mean \pm SEM. $p$ values $<0.05$ were considered significant.

\section{Results}

\section{Myo $\mathrm{X}$ regulating axonal initiation and contralateral branching/targeting in developing cerebral cortex}

To investigate the function of Myo $\mathrm{X}$ in axon development in vivo, we used Cre-LoxP recombination technology in combination with IUE to delete Myo X in portions of projection neurons in developing neocortex. Specifically, we examined the axon development of upper layer (L2-L4) neurons that project within the cortex, mostly via the corpus callosum (CC), and have birthdays at E14.5 to E15.5 (Kwan et al., 2012). IUE was thus performed at these time points, and the three key processes of axon development, including axon initiation, growth, and branching/ 

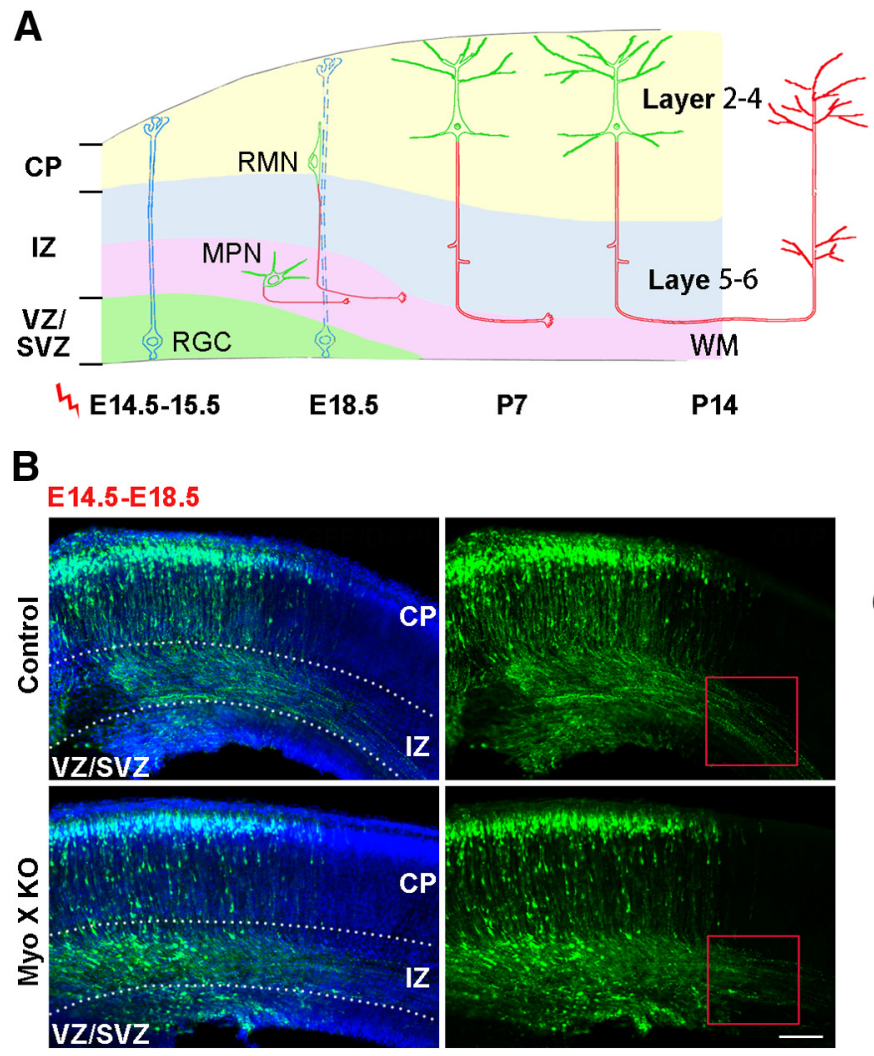

C

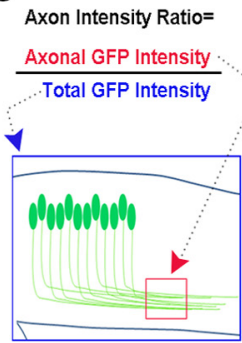

E

E

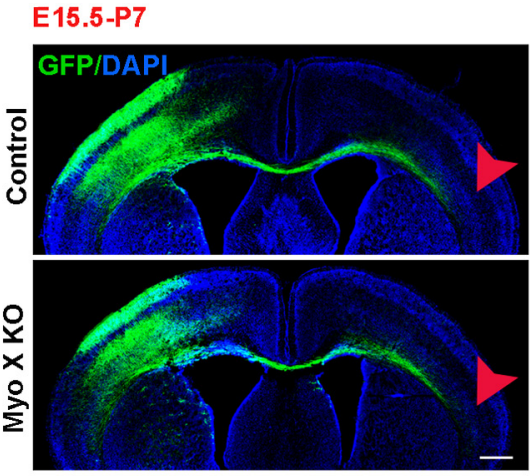

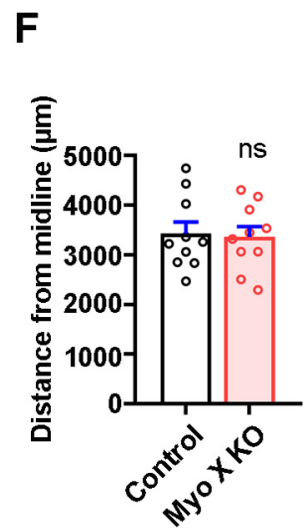

G

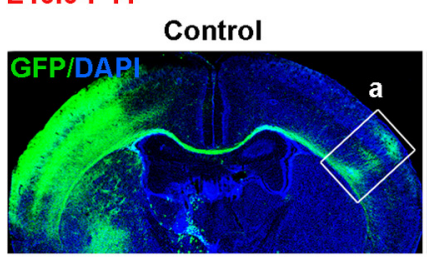

a

b

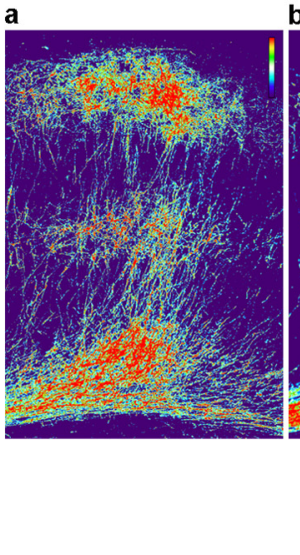

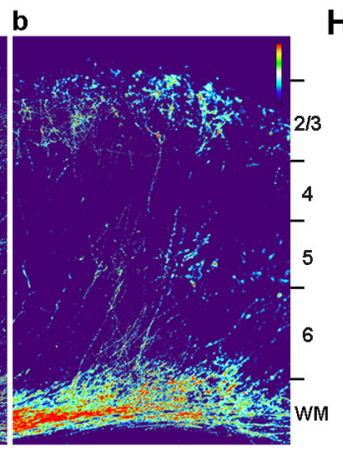

Myo X KO

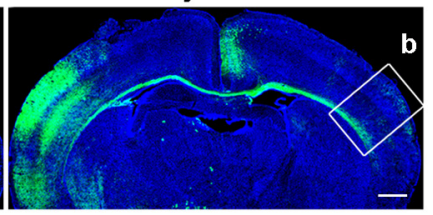

H

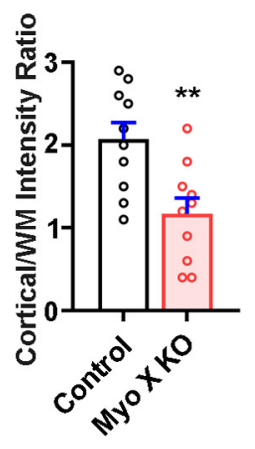

Figure 1. Defective axon initiation and contralateral branching in Myo X-K0 cortical neurons. $\boldsymbol{A}$, Schematic diagram of IUE assay for axon development in neocortex. $\boldsymbol{B}-\boldsymbol{D}$, E18.5 cerebral cortex that were electroporated with Cre-GFP plasmids into Myo Xf/f embryos or WT embryos at E14.5. $\boldsymbol{B}$, Representative images. Scale bar, $100 \mu \boldsymbol{m}$. $\boldsymbol{C}$, Quantification of axon initiation by using the axon intensity ratio, which is defined as axonal GFP intensity (marked by a red square) over the total GFP intensity (marked as a blue square), as illustrated in the schematic diagram. Student's $t$ test, $p=0.0379$. D, Quantification of GFP-positive cells in CP. Student's $t$ test, $p<0.0001$. E, F, P7 cerebral cortex that were electroporated with GFP (Control) or Cre-GFP (Myo XK0) plasmids into Myo Xf/f embryos at E15.5. $\boldsymbol{E}$, Representative images. Scale bar, $500 \mu \mathrm{m}$. $\boldsymbol{F}$, Quantifications of axon elongation. Student's $t$ test, $p=0.84$. $\boldsymbol{G}, \boldsymbol{H}, \mathrm{P} 14$ cerebral cortices that were electroporated with GFP (Control) or Cre-GFP (Myo X-KO) plasmids into Myo X/f embryos at E15.5. G, Representative images are shown. Scale bar, $500 \mu \mathrm{m}$. $\boldsymbol{H}$, Quantifications of axon intensity in the contralateral cortex (as indicated by a and $b$ in $G$ ). Student's $t$ test, $p=0.0044$. Data are presented as the mean \pm SEM. The numbers of brain sections scored are from three different brains for each group and indicated on the graphs. ns, No significant difference; $* p<0.05 ; * * p<0.01 ; * * * p<0.001$.

targeting, were evaluated as illustrated in Figure $1 A$. To determine the function of Myo X in axon initiation, Cre-GFP was electroporated into the neocortex of Myo $\mathrm{X}^{\mathrm{f} / \mathrm{f}}$ and wild-type (WT) embryos at E14.5, and their axon intensity ratio (Namba et al., 2014) was analyzed at E18.5, a critical time window for axon initiation (Namba et al., 2015). As shown in Figure 1, $B$ and $C$, Myo X knockout $(\mathrm{KO})$ resulted in a reduction in the axon intensity ratio in the brain, suggesting a role of Myo $\mathrm{X}$ in this event. In addition, neuronal migration appeared to be impaired (Fig. $1 B$, $C)$, as reported previously using RNA interference technology (Lai et al., 2015). We then evaluated axonal growth and midline crossing in control and MyoX-KO cortical neurons. MyoX-KO cortical neurons were achieved by IUE of Cre-GFP or GFP (as a control) into the neocortex of Myo $\mathrm{X}^{\mathrm{f} / \mathrm{f}}$ embryos (at E15.5; Wang et al., 2019); and the electroporated brain samples were examined at P7, a critical time window for cortical neuronal axon growth and midline crossing (Tagawa and Hirano, 2012; Fenlon and Richards, 2015). To our surprise, axons of Myo X-KO (Cre$\mathrm{GFP}^{+}$) neurons crossed the midline, and their lengths were comparable to those of control axons (Fig. $1 E, F$ ), suggesting little role, if there is any, for Myo X to play in axon growth or middling crossing. We finally examined axon contralateral branching/targeting in P14 control and Myo X-KO cortical brains. As shown in Figure 1, $G$ and $H$, axon branching/targeting to the contralateral cortex was detected in control neurons; but, this process was largely impaired in Myo X-KO neurons. These 
results suggest necessity of Myo X in promoting axon branching/ targeting. Together, these results reveal unrecognized roles of Myo $\mathrm{X}$ in axon initiation and contralateral branching/targeting in developing cerebral cortex.

\section{Netrin-1 promoting Myo X-regulated axonal initiation and branching}

Given the specific orientation of the trailing processes or nascent axons, we speculate that extracellular signals from ventricular zone (VZ) and subventricular zone (SVZ) modulate intrinsic signaling (e.g., Myo X) for axon initiation and development, and Netrin-1, an upstream Myo X regulator (Zhu et al., 2007), may be involved in axon initiation and branching. To test this speculation, we examined the function of Netrin-1 in axon development by IUE of Cre-GFP into Netrin-1 floxed $\left(\mathrm{NTN1}^{\mathrm{f} / \mathrm{f}}\right)$ embryos at E14.5. The electroporated brain samples were examined at E18.5 to evaluate the neuronal axon intensity ratio. Indeed, a reduction in the axon intensity ratio of cortical neurons was detected in Netrin-1-KO embryos (Fig. 2A,B), revealing a similar role of Netrin-1 as that of Myo $\mathrm{X}$ in axon initiation. Neuronal migration was not affected by Netrin-1 KO (Fig. $2 \mathrm{~A}$, $C)$. We next asked whether Netrin-1-regulated axon initiation depends on Myo X. To this end, plasmids encoding Myc-Netrin1 and Myo X miRNA were coelectroporated into the E14.5 embryos (Fig. 2D). Netrin-1 ectopic expression restored the axon intensity ratio in Myo X-KD neurons (Fig. 2D,E), supporting the view for Netrin-1-Myo $\mathrm{X}$ pathway in promoting axon initiation.

We then asked whether the Netrin-1-DCC pathway plays a role in axon branching/targeting similar to that of Myo X. Netrin-1 or DCC expression in E15.5 cortical neurons were suppressed by IUE of their shRNAs, respectively. The knock-down efficiency of Netrin-1 or DCC shRNA was examined by Western blot (Fig. $2 F, G$ ). Their axons at the age of P14 were examined. Netrin-1 KD in E15.5 cortical neurons had little effect on the axonal contralateral branching/targeting (Fig. 2H,I). However, DCC KD impaired axonal contralateral growth and branching (Fig. $2 H, J)$. These results, which are in line with our hypothesis, suggest that DCC and Myo X in neurons are necessary to promote axon branching, but Netrin-1, an extracellular cue, regulates axon development in cell-nonautonomous fashion.

\section{Netrin-1 increasing axonal distribution and transport of Myo X in cultured neurons}

To understand how Netrin-1 regulates the function of Myo X in axon development, we examined the effect of Netrin-1 on exogenous Myo X (GFP-Myo X) distribution in cultured neurons. To our surprise, GFP-Myo X was largely distributed in the soma and the tips of dendrite-like filopodia, but was low in Tau-1-positive axonal compartments in the absence of Netrin-1 (Fig. 3A). Upon Netrin-1 stimulation, an obvious increase of GFP-Myo X in Tau-1-positive axons with a slight decrease of GFP-Myo X in MAP2-positive dendritic neurites were detected (Fig. 3A-D), suggesting a role of Netrin-1 in regulating GFP-Myo X distribution in axons and dendrites.

Next, we examined the dynamics of GFP-Myo X in axons by FRAP (Fig. 3E). At $3 \mathrm{~d}$ in vitro (DIV3), neurons exhibited a type of polarized morphology with a very long axon and multiple short dendrites. The longest neurite, likely to be the axon (Dotti et al., 1988), was analyzed. The fluorescence recovery of GFPMyo X in control axons was much slower and more incomplete than that of Netrin-1-treated axons (Fig. $3 E-G$ ), supporting the view for Netrin-1 to enhance GFP-Myo X movement. In contrast to axonal GFP-Myo X, GFP-Myo X in dendrite-like filopodia exhibited a puncta pattern (Fig. $3 H$ ). Time-lapse imaging and analyzing the motility of GFP-Myo X in these filopodia showed both extension and retraction movements of GFP-Myo X puncta in control neurons (Fig. $3 H$, bottom panels). Upon Netrin-1 stimulation, the traveling path and the average velocity of GFPMyo X puncta were all reduced (Fig. $3 H$, bottom panels, $I$ ), with an increase in the percentage of stationary puncta (Fig. 3J). Since Myo X motility is coupled with the filopodia movement and MyoX undergoes forward and reverse movement along filopodia (Berg and Cheney, 2002), it is possible that Netrin-1 suppressed both motilities of GFP-MyoX and filopodia. Together, these results suggest that while Netrin-1 increases anterograde movement of GFP-Myo X in axons, it decreases the motility of GFPMyo X in dendrite-like filopodia.

\section{Requirement of DCC and PI3K activation for Netrin-1 increased axonal distribution of Myo X}

To further understand how Netrin-1 regulates the axonal distribution of Myo X, we first mapped domains in GFP-Myo X that are necessary for this event. Myo $\mathrm{X}$ is a multidomain containing unconventional myosin, containing $3 \mathrm{PH}$ domains, an MyTh4 domain, and a band in the FERM domain in the $\mathrm{C}$ terminus, in addition to the motor domain in the $\mathrm{N}$ terminus (Berg et al., 2000; Kerber and Cheney, 2011). GFP-Myo X deletion mutants were generated as illustrated in Figure $4 A$ (left). In the absence of Netrin-1, Myo X mutants containing the motor domain showed a distribution pattern similar to that of full-length Myo X, with predominant localizations in the soma and dendritic filopodia (Fig. $4 A, B$ ). However, in the presence of Netrin-1, Myo X deletions in the second $\mathrm{PH}$ domain $\left(\mathrm{Myo} \mathrm{X}^{\Delta \mathrm{PH} 2}\right.$ ) or in the FERM domain (Myo $\mathrm{X}^{\triangle \mathrm{FERM}}$ ) abolished Netrin-1-induced axonal distribution (Fig. $4 A, B$ ). These results suggest a requirement of both $\mathrm{PH}$ and FERM domains in Myo X for Netrin-1-induced axonal distribution of Myo X.

As the FERM domain in Myo X binds to DCC (Zhu et al., 2007), we thus speculate that DCC-Myo X interaction may be critical for this event. Indeed, this view is supported by the observations that DCC-Myo X interaction is upregulated by Netrin-1 (Fig. 5A,B), and neurons suppressing DCC expression by its shRNA failed to response to Netrin-1 in targeting Myo X to axons (Fig. 5C-E).

$\mathrm{PH}$ domains of Myo X are known to bind to multiphosphoinositols, including PI(4,5)P2, PI(3,4)P2, and PI(3,4,5)P3 (Cox et al., 2002; Umeki et al., 2011). The second PH domain is crucial for binding to $\mathrm{PI}(3,4,5) \mathrm{P} 3 / \mathrm{PI}(3,4) \mathrm{P} 2$, products of $\mathrm{PI} 3 \mathrm{~K}$ activation (Umeki et al., 2011). As this PH domain in Myo X is necessary for Netrin-1-induced axonal distribution of Myo X, we wondered whether $\mathrm{PI}(3,4,5) \mathrm{P} 3 / \mathrm{PI}(3,4) \mathrm{P} 2$ are involved in the axonal distribution of Myo X. To this end, neurons expressing GFPMyo X were treated with Netrin-1 in the presence or absence of wortmannin $(10 \mathrm{nM})$, an inhibitor of PI3K that blocks the production of $\mathrm{PI}(3,4,5) \mathrm{P} 3$ and $\mathrm{PI}(3,4) \mathrm{P} 2$. Such an inhibition abolished Netrin-1-induced axonal distribution of Myo X (Fig. $5 \mathrm{~F}-\mathrm{H})$, supporting a role for $\mathrm{PI} 3 \mathrm{~K}$ activation and its products, $\mathrm{PI}$ $(3,4,5) \mathrm{P} 3 / \mathrm{PI}(3,4) \mathrm{P} 2$, in this event.

\section{Myo X interaction with KIF13B, a kinesin family motor protein}

To further investigate the underlying mechanisms of Netrin-1stimulated Myo X anterograde transport, we attempted to identify additional Myo X binding proteins by use of the LC-MS/MS to sequence the proteins associated with the Myo X immunoprecipitates. Specifically, GFP-hMyoX (GFP-tagged headless MyoX) 
A

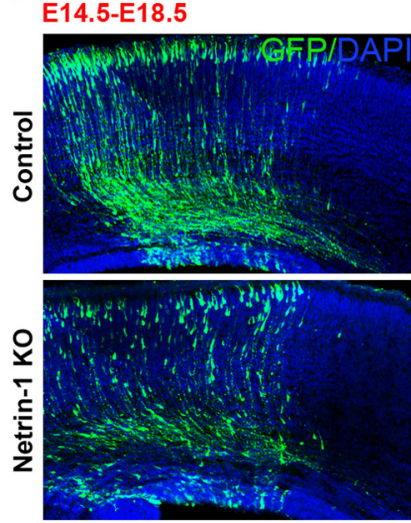

B

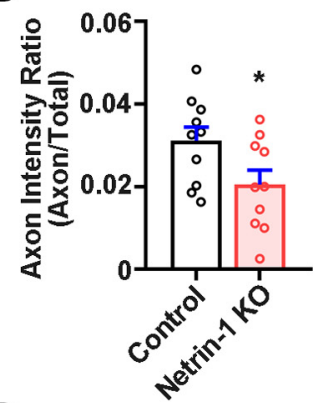

D
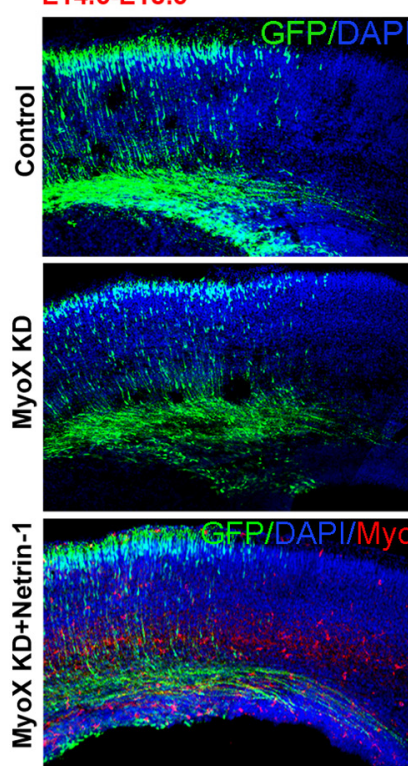

F

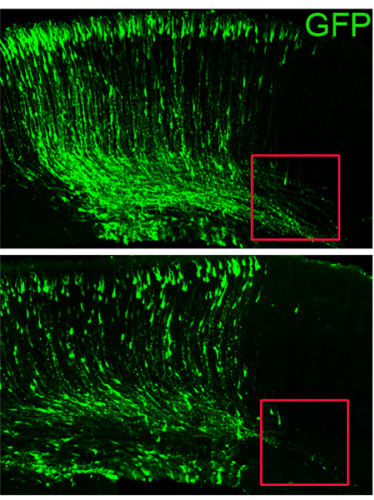

C
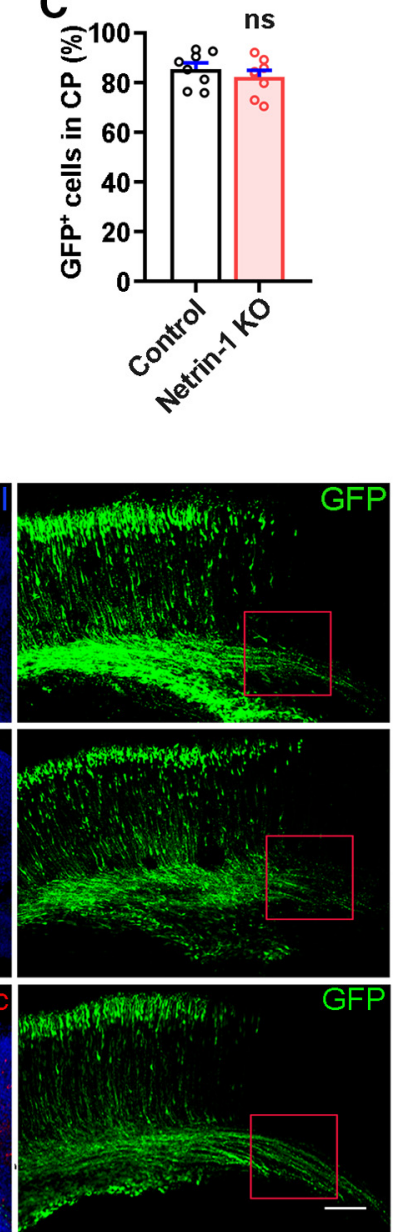

H
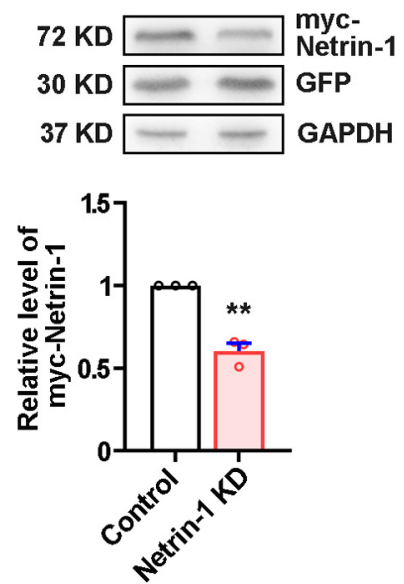

E15.6-P14

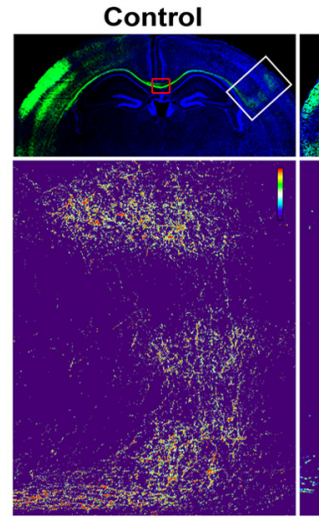

Netrin-1 KD
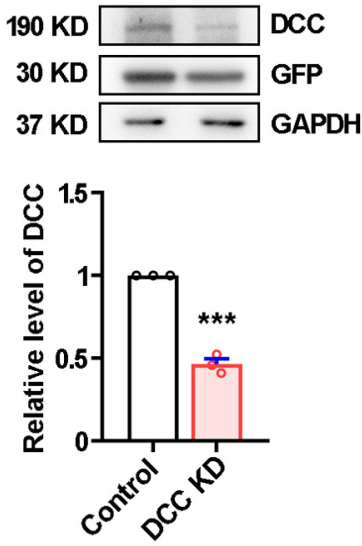
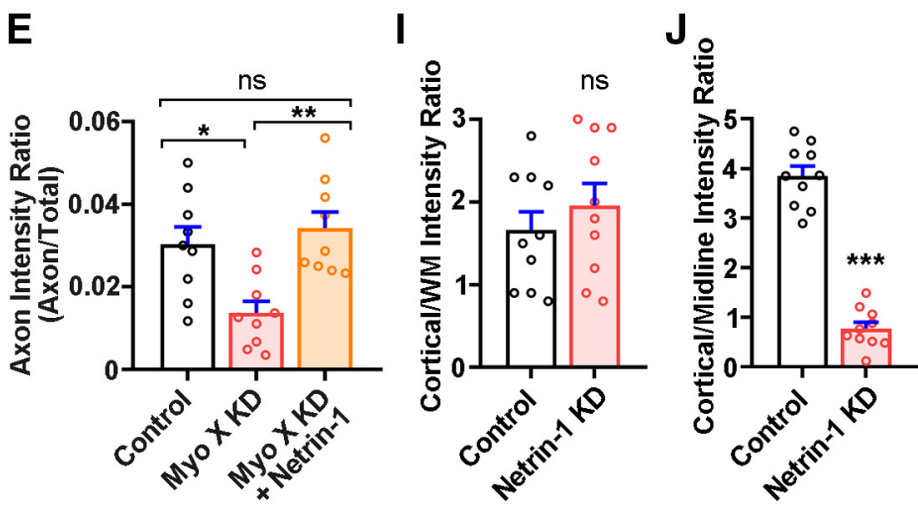

Figure 2. Netrin-1 rescue of axon initiation defect caused by Myo X deficiency. A-C, E18.5 cerebral cortices that were electroporated with GFP (Control) or Cre-GFP (Netrin-1-K0) plasmids into NTN1 ${ }^{\mathrm{f} / \mathrm{f}}$ embryos at E14.5. $\boldsymbol{A}$, Representative images. Scale bar, $100 \mu \mathrm{m}$. B, Quantification of axon intensity ratio. Student's $t$ test, $p=0.0418$. C, Quantification of GFP ${ }^{+}$cells in CP. Student's $t$ test, $p=0.39$. D, Representative images of E18.5 cerebral cortex electroporated with control miRNA (Control), Myo X miRNA (Myo X KD), or Myo X miRNA together with MycNetrin-1 plasmids (Myo X KD + Netrin-1) at E14.5. Scale bar, $100 \mu \mathrm{m}$. $E$, Quantification of axon intensity ratio. One-way ANOVA: $p=0.0303$ for the MyoX KD group; $p=0.74$ for Myo $X$ KD + Netrin-1 group. $\boldsymbol{F}, \boldsymbol{G}$, Immunoblotting analysis of Netrin-1-KD and DCC-KD efficiency. $\boldsymbol{F}$, HEK293T cells were transfected with a scrambled or Netrin-1 shRNA together with Myc-Netrin-1 plasmids for $48 \mathrm{~h}$. Cell lysates were extracted for Myc immunoblotting. Student's $t$ test was used for the statistical analysis of Netrin-1-KD efficiency, $p=0.0012$. G, NLT cells were transfected with a control or DCC miRNA for $48 \mathrm{~h}$. Cell lysates were extracted for DCC immunoblotting. Student's $t$ test was used for the statistical analysis of Netrin-1-KD efficiency, $p<0.0001$. $\boldsymbol{H}$, Representative images of P14 cerebral cortex electroporated with Control miRNA (Control), Netrin-1 shRNA (Netrin-1 KD), and DCC miRNA (DCC KD) at E15.5. Scale bar, $500 \mu \mathrm{m}$. $I$, Quantification of axon contralateral branching. Student's $t$ test, $p=0.39$. J, Quantification of axon contralateral branching by using the cortical/midline intensity ratio, which is defined as axonal GFP intensity (marked by a white rectangle) over the midline GFP intensity (marked as a red rectangle), as illustrated in the schematic diagram. Student's $t$ test, $p<0.0001$. Data are presented as the mean \pm SEM. The numbers of brain sections scored are from three different brains for each group and indicated on the graphs. ns, No significant difference; $* p<0.05 ; * * p<0.0$; $* * * p<0.001$. 
A

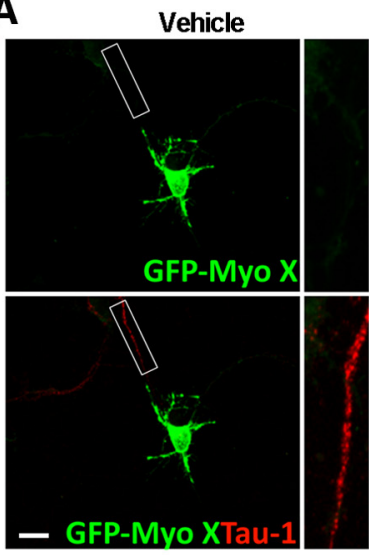

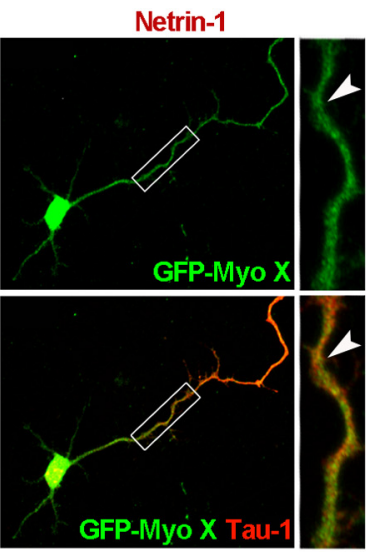

F
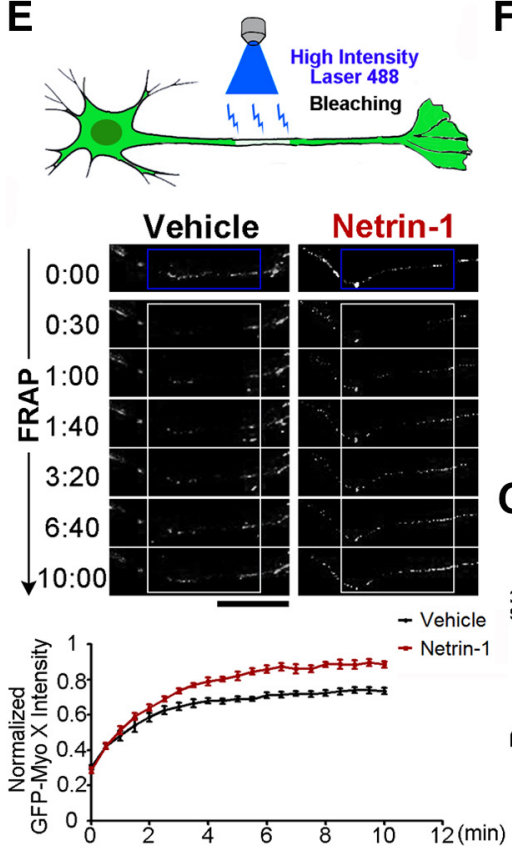

B

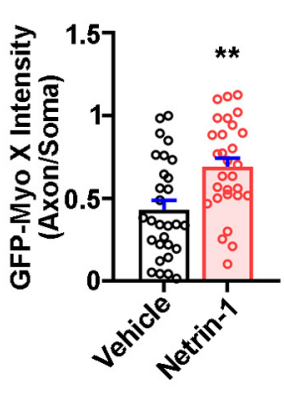

C

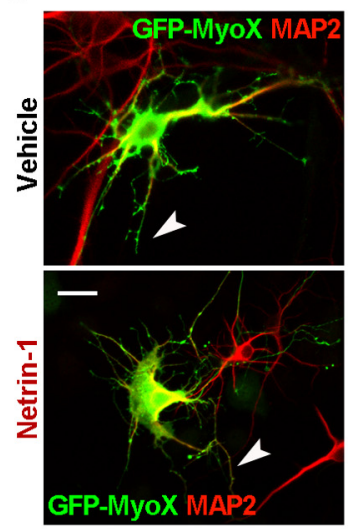

D

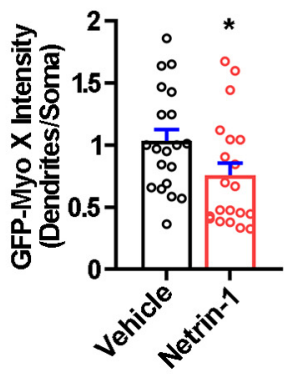

H

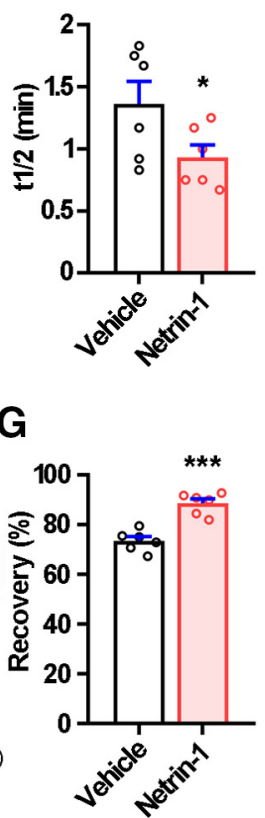

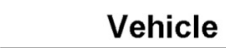
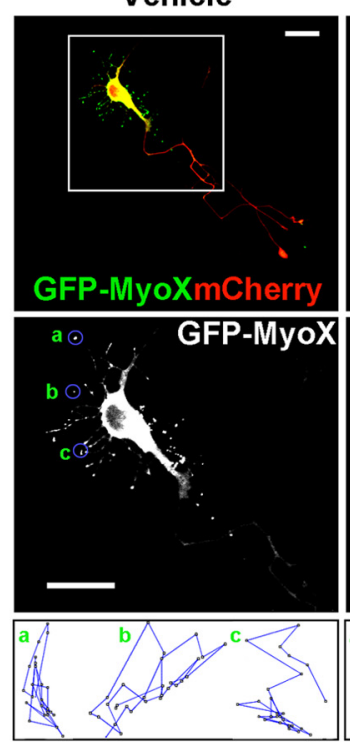

Netrin-1
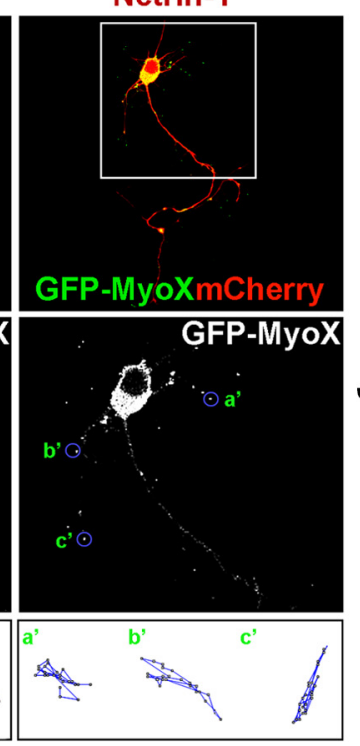

I

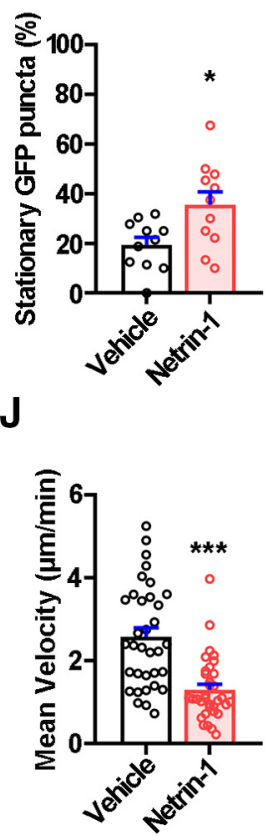

Figure 3. Increase of axonal distribution and anterograde transport of GFP-Myo $X$ by Netrin-1. A-D, Immunostaining analysis using indicated antibodies at DIV3 neurons that were transfected with GFP-Myo $X$ at DIV1 and treated with vehicle or Netrin- 1 for $1 \mathrm{~h}$. $A$, Images marked by rectangles were amplified and shown in the right panels. White arrowheads indicate the axonal distribution of GFP-Myo X. Scale bar, $10 \mu \mathrm{m}$. B, Quantification of GFP-Myo X intensity in axons. The axonal GFP-Myo X level was normalized to somatic GFP-Myo X. Student's $t$ test, $p=0.0012$. C, Yellow arrows indicate GFP-Myo X distribution in MAP2-positive dendrites. Scale bar, $10 \mu \mathrm{m}$. D, Quantification of GFP-Myo X intensity in dendrites. The dendritic GFP-Myo X level was normalized to somatic GFP-Myo X. Student's $t$ test, $p=0.0471$. $\boldsymbol{E}$-G, Analysis of axonal GFP-Myo X mobility with FRAP assay. $\boldsymbol{E}$, Images of FRAP analysis in GFP-Myo X-expressing neurons in the presence of vehicle or Netrin-1 and the quantification (normalized GFP-Myo X intensity of the photobleached axon compartment). Scale bar, $20 \mu \mathrm{m}$. $\boldsymbol{F}$, Quantification of half-time of maximum recovery (t1/2). Student's $t$ test, $p=0.0446, n=6$ neurons from three different experiments. G, Percentage of GFP-Myo X recovery. Student's $t$ test, $p<0.0001, n=6$ neurons from three different experiments. $\boldsymbol{H}-\boldsymbol{J}$, Time-lapse imaging analysis of GFP-Myo X-expressing neurons in the presence of vehicle or Netrin-1. $\boldsymbol{H}$, the mCherry was cotransfected to visualize neuronal processes. Images marked in rectangular were amplified and showed in the middle panels. Mobile trajectory of indicated GFP-Myo X puncta was presented in the bottom panels. Scale bar, 20 $\mu \mathrm{m}$. I, Quantification of mean velocity of GFP-Myo X puncta. Student's $t$ test, $p<0.0001$. J, Quantification of stationary GFP-Myo X. Student's $t$ test, $p=0.0146$. Data are presented as the mean \pm SEM. The numbers of neurons scored are from 3 different experiments for each group and indicated on the graphs. $* p<0.05 ; * * p<0.01 ; * * * p<0.001$.

was transfected into cultured cortical neurons. The cell lysates were immunoprecipitated with anti-IgG (as a control) or antiGFP antibody. The immune complexes were resolved by SDS-PAGE and subjected to silver staining. All specific bands were subjected to LC-MS/MS. Five hundred eightysix proteins were detected in the immunoprecipitates with anti-GFP antibody, including previously reported binding partners, such as actin, $\alpha$-tubulin, Arp $2 / 3$ complex, and E-cadherin (Fig. 6A). Interestingly, KIF13B (polypeptide amino acid sequence, LSLVDLAGSER, TVAATNMNEESSR, GSLLSEPAIQVR, DVPTGGIFQLR; accession \#E9Q4K7) was identified (Fig. 6A). Given that anterograde transport is powered by kinesin family motor (Hirokawa et al., 2010), we thus asked whether Myo X acted as a cargo of KIF13B to be transported along microtubules in axons. It is of interest to note the report that KIF13B is essential for anterograde transport of $\mathrm{PI}(3,4,5) \mathrm{P} 3$ for axonal outgrowth and formation (Yoshimura et al., 2010). We then mapped the domains in KIF13B for its interaction with Myo X by coimmunoprecipitation assay. KIF13B contains a motor domain at the NH2 terminus, a FHA (forkhead-associated) domain, a MAGUK binding stalk domain, two domains of unknown 
A

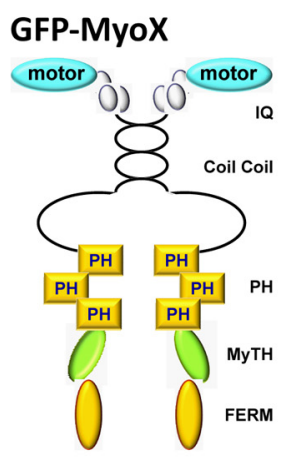

GFP-MyoX $\Delta$ PH2

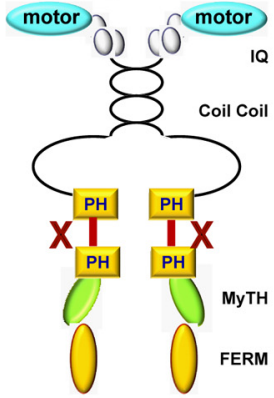

GFP-MyoX $\Delta$ FERM motor

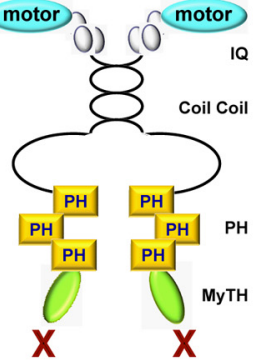

Control
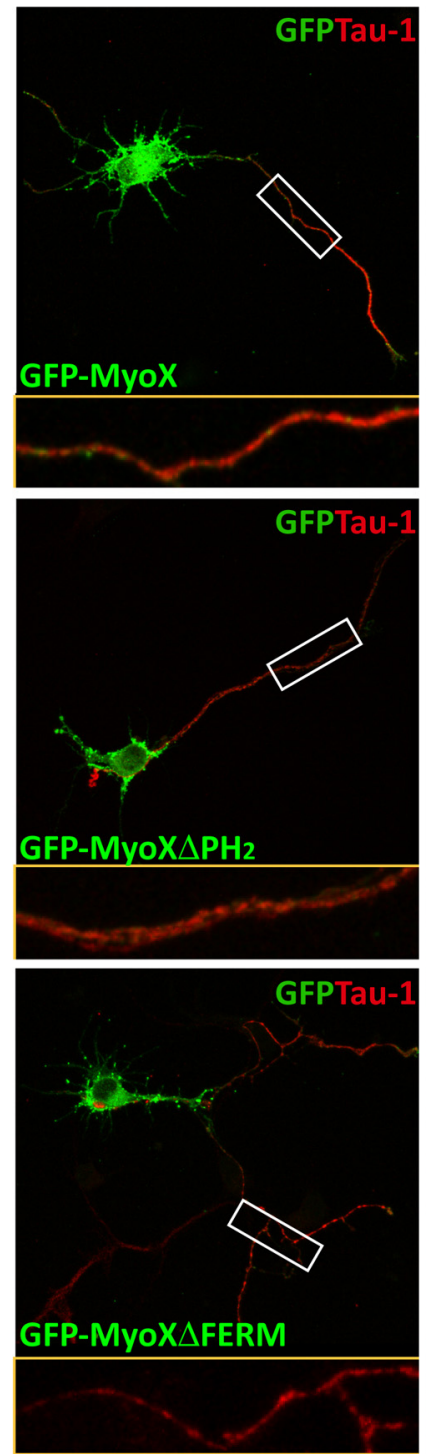

B

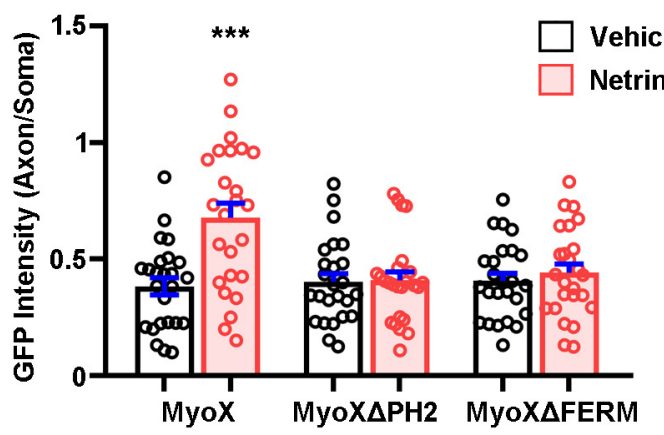

Netrin-1
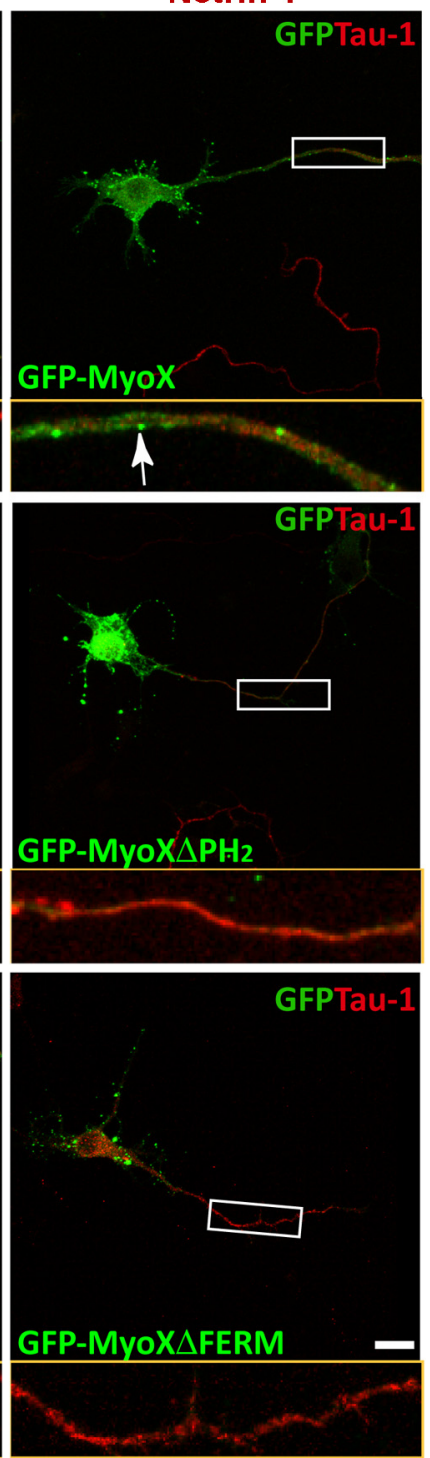

\section{Vehicle}

Netrin-1

Figure 4. Requirement of the PH and FERM domains of Myo X for Netrin-1 increase of axonal distribution of exogenous GFP-Myo X. A, Neurons transfected with GFP-Myo $X$ and its deletion mutants (illustrated in the left panels) were treated with vehicle or Netrin-1 for $1 \mathrm{~h}$ and then stained with anti-Tau- 1 antibody at DIV3. Scale bar, $10 \mu \mathrm{m} . \boldsymbol{B}$, Quantification of axonal distribution of GFP-Myo X or its deletion mutants. Student's $t$ test: for Myo X group, $p=0.0003$; for Myo $\Delta$ PH group, $p=0.83$; for Myo $\Delta$ FERM group, $p=0.46$. Data are presented as the mean \pm SEM. The numbers of neurons scored in these groups are from three different experiments and are indicated on the graphs. ns, № significant difference; $* * * p<0.001$.

function and a CAP-Gly motif at the C terminus (Fig. 6B). As shown in Figure 6, $B$ and $C$, terminal regions of KIF13B (Myc tagged; including KIF13B ${ }^{558-1826}$, KIF13B ${ }^{990-1826}$, and KIF $13 \mathrm{~B}^{1532-1826}$ ), but not the $\mathrm{N}$ terminus (KIF13B ${ }^{1-557}$ ), were detected in Myo X immunoprecipitates. Further analysis of their interaction identified that the C-terminal domain KIF13B $\mathrm{B}^{1532-1826}$ is involved in the interaction with Myo X.

The Myo X-KIF13B interaction was further verified by a GST pulldown assay. The recombinant GST-KIF13B ${ }^{1532-1826}$ fusion protein was produced (Fig. $6 \mathrm{C}$ ), which was used to pull down lysates expressing various GFP-Myo X mutants (including Myo 
A

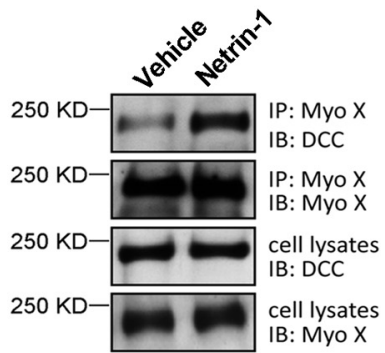

C

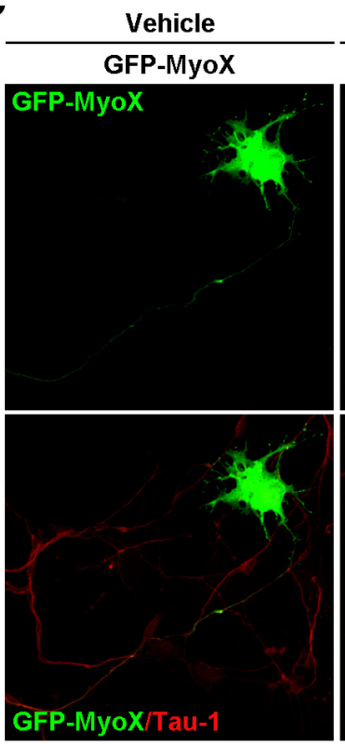

$\mathbf{F}$

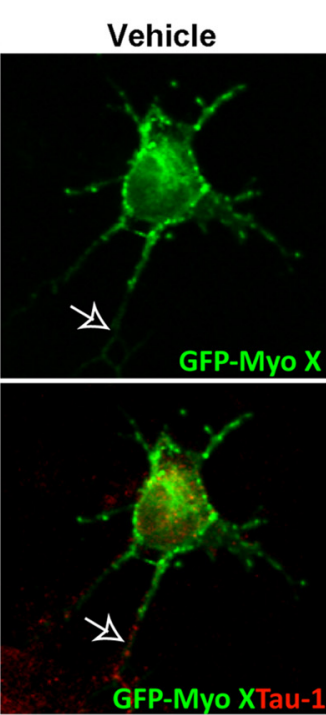

B

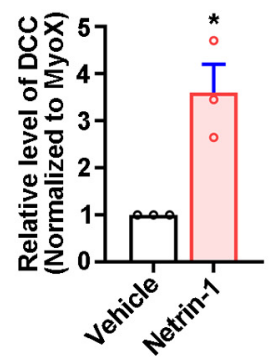

D

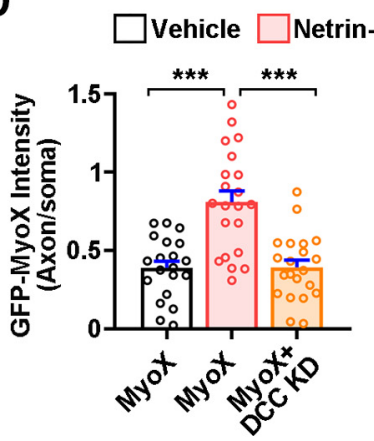

Netrin-1

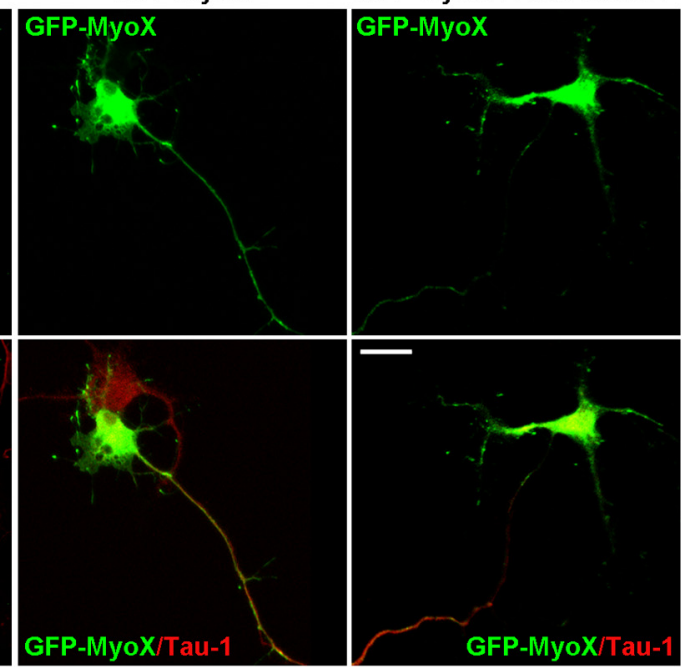

E
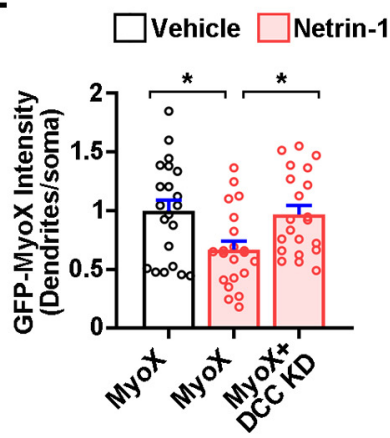

G

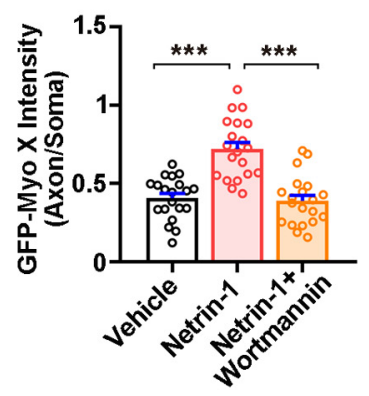

H
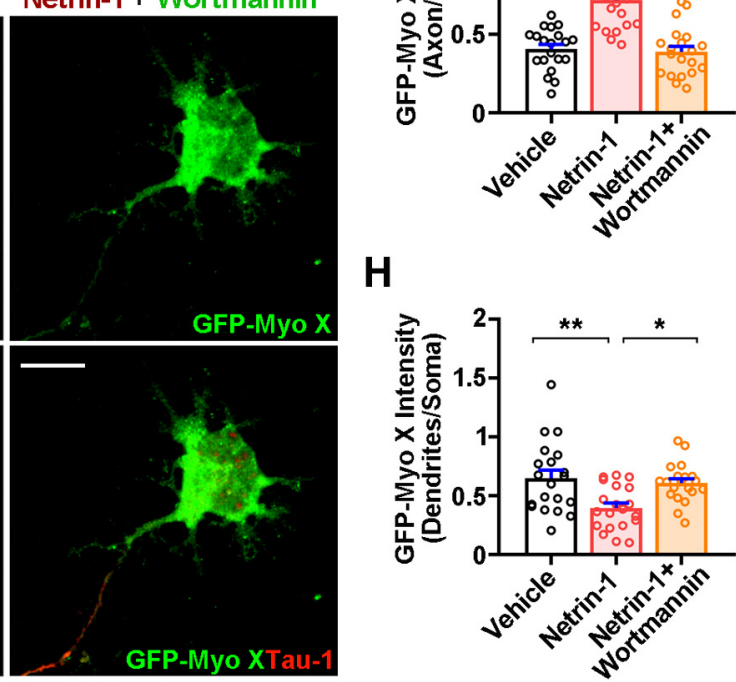

Figure 5. $D C C$ and PI3K activity-dependent axonal distribution of GFP-Myo $X$ in response to Netrin-1 stimulation. $A$, Immunoprecipitation analysis of DCC association with Myo $X$ in cultured cortical neurons that were treated with vehicle or Netrin-1 for $1 \mathrm{~h}$. B, Quantifications of DCC binding with MyoX. Student's $t$ test, $p=0.0122$. C, Neurons transfected with GFP-Myo X or GFPMyo $X$ together with DCC miRNA were treated with vehicle or Netrin-1 and then stained with anti-Tau-1 antibody at DIV3. Scale bar, $10 \mu$ m. $D$, Quantification of axonal distribution of GFPMyo X. One-way ANOVA, for Myo $X$ group with vehicle stimulation and Myo $X$ group with Netrin-1 stimulation, $p<0.0001$; for Myo $X$ and Myo $X+D C C-K D$ groups, $p<0.0001$. E, Quantification of dendritic distribution of GFP-Myo X. One-way ANOVA, for Myo $X$ groups with vehicle and Myo $X$ group with Netrin- 1 stimulation, $p=0.0142$, for Myo $X$ and Myo $X+D C C-$ KD groups, $p=0.0283$. $F$, GFP-Myo X-expressing neurons were treated with vehicle and Netrin-1 with or without PI3 kinase inhibitor, wortmannin (10 nM) for $1 \mathrm{~h}$ and subjected to immunostaining with anti-Tau-1 antibody at DIV3. Scale bar, $10 \mu \mathrm{m}$. G, Quantification of axonal distribution of GFP-Myo X. One-way ANOVA, $p<0.0001$ for both groups. $\boldsymbol{H}$, Quantification of dendritic distribution of GFP-Myo X. One-way ANOVA, for vehicle and Nerin-1 groups, $p=0.0023$; for Netrin-1 and Netrin-1+ wortmannin groups, $p=0.0124$. Data are presented as the mean \pm SEM. The numbers of brain sections scored are from three different brains for each group and indicated on the graphs. ns, No significant difference; $* p<0.05 ; * * p<0.01 ; * * * p<0.001$. 


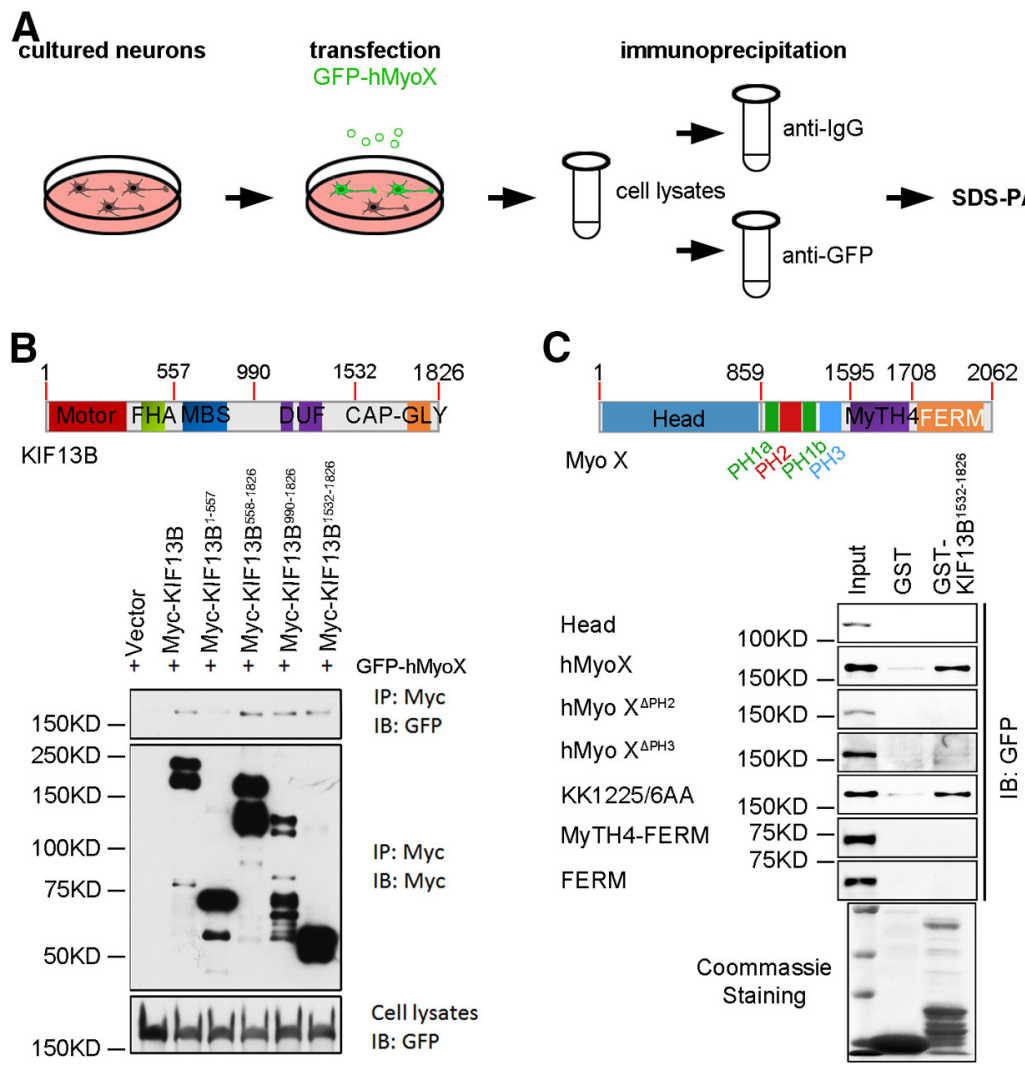

E
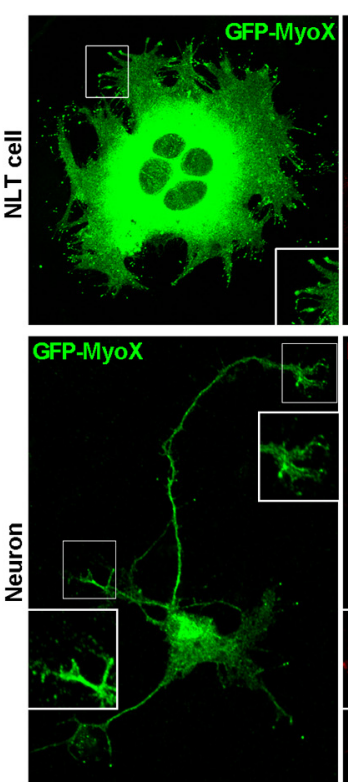
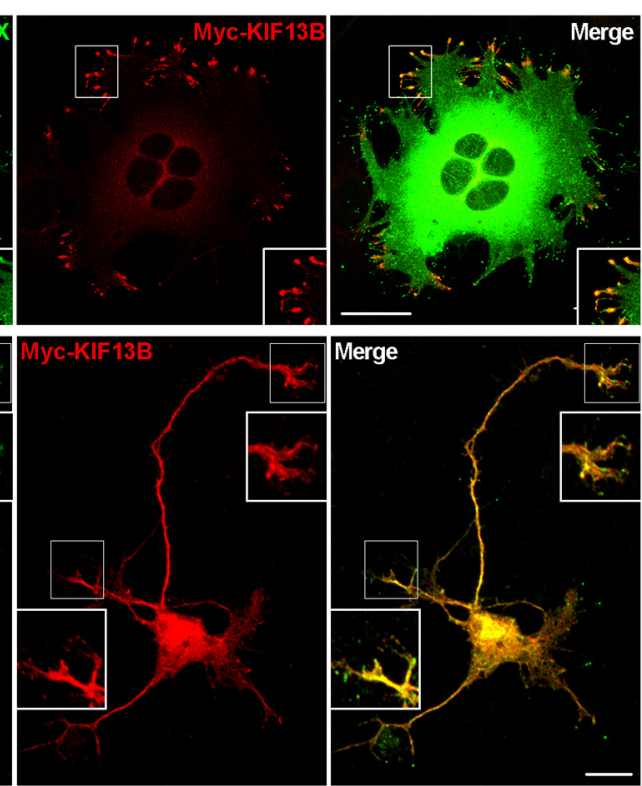

D

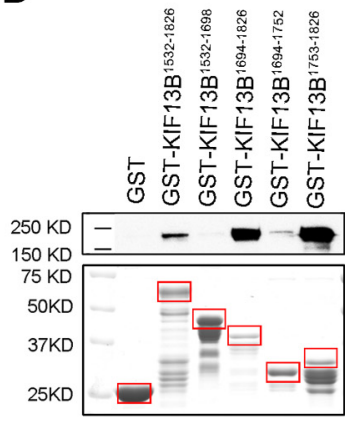

Coommassie Staining

F
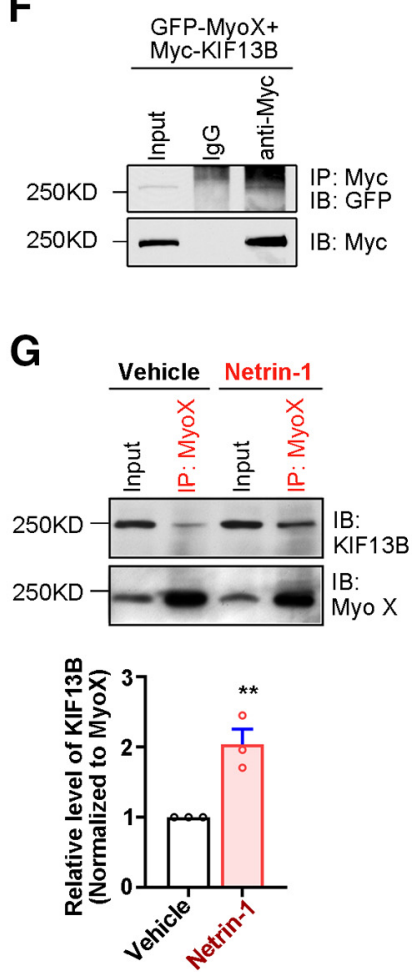

Figure 6. Interaction of Myo X with KIF13B. A, KIF13B was identified as a binding partner of MyoX by LC-MS/MS. B, Coimmunoprecipitation of GFP-hMyo X and Myc-KIF13B ${ }^{1532-1826}$. GFPhMyo X was coexpressed with Myc-KIF13B and its deletion mutants in HEK293T cells and immunoprecipitated by anti-Myc antibody. $C$, Immunoblotting of the pulled down fraction by the GST-KIF13B C-terminus fusion protein and GST alone. The numbers of GST fusion proteins and GST were revealed by Coomassie blue staining (bottom). D, Immunoblotting of the pulled down fraction by the truncated C-terminal domains fused with GST and GST alone. The amounts of different GST fusion proteins and GST alone were revealed by Coomassie blue staining (bottom). $\boldsymbol{E}$, Colocalization of GFP-Myo X and Myc-KIF13B in filopodia tips of NLT cells (top panels) and in axon and dendrite-like filaments in cultured cortical neurons (bottom panels). Images marked in rectangular was amplified and included as insert. Scale bar, $20 \mu m$. $\boldsymbol{F}$, Coimmunoprecipitation of exogenous Myc-KIF13B and GFP-Myo X. HEK293T cell lysates were immunoprecipitated with anti-Myc antibody and with IgG as control. $\mathbf{G}$, Immunoprecipitation of endogenous Myo X and KIF13B with or without Netrin-1 stimulation. Neurons treated with vehicle or Netrin-1 were lysed and incubated with anti-Myo X antibody (top). Quantifications of KIF13B binding with MyoX are presented in the bottom panel. Student's $t$ test, $p=0.0089$. Data are presented as the mean \pm SEM. For statistical analysis, three independent experiments were performed and indicated on the graphs. $* * p<0.01$. 
$\mathrm{X}^{\text {Head }}$, hMyo X, hMyo $\mathrm{X}^{\Delta \mathrm{PH} 2}$, hMyo $\mathrm{X}^{\Delta \mathrm{PH} 3}$, hMyo $\mathrm{X}^{\mathrm{KK} 1225 / 6 \mathrm{AA}}$, Myo $X^{\text {Myth4-Ferm }}$, and Myo $\left.\mathrm{X}^{\text {Ferm }}\right)$. hMyoX is an abbreviation of headless MyoX, which contains amino acids from 860 to 2062. $\mathrm{hMyoX}^{\mathrm{KK} 1225 / 6 \mathrm{AA}}$ means that the $1225 / 1226$ lysine was further mutated to alanine. These two lysines are located in the second $\mathrm{PH}$ domain and are required for Myo X binding with $\mathrm{PI}(3,4,5)$ P3. Note that only hMyo X and hMyo $\mathrm{X}^{\mathrm{KK} 1225 / 6 \mathrm{AA}}$ were pulled down by GST-KIF13B ${ }^{1532-1826}$, suggesting the requirement of the second and third $\mathrm{PH}$ domains of Myo $\mathrm{X}$ for its binding to the C-terminal domain in KIF13B (Fig. 6C). By this assay, the effective Myo X binding region in KIF13B was further mapped to the last 74 aa in its $C$ terminus (Fig. $6 D$ ). It is noteworthy that while the site KK1225/6 in Myo X is critical for binding to PI $(3,4,5) \mathrm{P} 3$ (Plantard et al., 2010), it was not required for Myo X interaction with KIF13B.

Finally, we examined Myo X-KIF13B interaction by coimmunostaining analysis. As shown in Figure 6E, GFP-Myo X was colocalized with Myc-KIF13B in filopodia tips in NLT cells and in axon and dendrite-like filaments in cultured cortical neurons. In addition, their interaction was reconfirmed by coimmunoprecipitation analysis of exogenously expressed GFP-Myo X and Myc-KIF13B (Fig. 6F), as well as endogenous KIF13B with Myo $\mathrm{X}$ in primary neuronal lysates (Fig. 6G). Interestingly, Netrin-1 stimulation increased Myo X-KIF13B interaction in neurons (Fig. 6G). Together, these results suggest that Myo $\mathrm{X}$ interacts with KIF13B, implicating KIF13B in Netrin-1-induced Myo X distribution in axons.

\section{Myo X as a cargo of KIF13B for its axonal distribution}

To investigate the function of KIF13B in Netrin-1-induced Myo $\mathrm{X}$ anterograde transportation, we first examined whether the distribution of GFP-Myo X in Tau-1-positive axons was affected by $\mathrm{KIF} 13 \mathrm{~B}$ expression. Indeed, the expression of KIF13B was sufficient to increase the localization of GFP-Myo X in axons and decrease the localization of GFP-Myo $\mathrm{X}$ in dendrites in the $\mathrm{ab}$ sence of Netrin-1(Fig. 7A-C). In line with this view was the observation by the FRAP assay that the recovery of GFP-Myo X after photobleaching in axons was sped up by KIF13B expression (Fig. 7D-F). Furthermore, the effect of KIF13B on GFPMyo $\mathrm{X}$ distribution was examined by time-lapse imaging analysis. As shown in Figure 7G, GFP-Myo X puncta exhibited high motility in dendrite-like neurites. Such actin-based motility of GFP-Myo X was decreased in neurons coexpressing KIF13B (Fig. 7G-I).

Second, we determined whether KIF13B was necessary for Netrin-1-induced Myo X axonal distribution. A plasmid encoding KIF13B shRNA was generated, which selectively suppressed KIF13B expression (Fig. 8A,B). GFP-Myo X with KIF13B shRNA or control shRNA were cotransfected into neurons treated with or without Netrin-1. In control neurons (GFP-Myo $\mathrm{X}$ with control shRNA), the distribution of GFP-Myo X in axons was increased and in dendrites was decreased by Netrin-1 stimulation (Fig. 8C-E). However, such an increase of the axonal distribution of Myo X was abolished in neurons cotransfected with KIF13B shRNA (Fig. 8C,D). Moreover, KIF13B KD in embryonic mouse cortical neurons significantly suppressed GFP-Myo $\mathrm{X}$ distribution in axon-like neurites (Fig. 8F,G). Together, these results suggest that KIF13B is not only sufficient, but also necessary for Netrin-1-induced Myo X distribution in axons. In line with this view, the distribution of KIF13B in axons was increased by Netrin-1 (Fig. $8 H-J$ ).

\section{KIF13B, as Myo X, promoting axonal initiation and contralateral branching/targeting in developing cerebral cortex}

The important role of KIF13B in Netrin-1-induced Myo X axonal distribution led us to speculate that that KIF13B plays a role similar to that of Myo X in Netrin-1-induced axonal initiation and targeting in vivo. To test this speculation, KIF13B shRNA and Myo X miRNA were transported by IUE into the progenitor cells of cortical neurons in E14.5 mouse embryos, and their brain sections at E18.5 were examined (Fig. 9A). As shown in Figure 9, $A$ and $B, \mathrm{KIF} 13 \mathrm{~B}$ KD resulted in a decrease in axon intensity ratio in the cortical brains, an impairment in axon initiation similar to that of Myo X-KD neurons. In addition, the percentage of Myo X- or KIF13B-deficient neurons into CP was decreased compared with that of control neurons (Fig. 9A,C). Furthermore, we analyzed the polarization of neurons in IZ and defined the longest neurites as axons by the following two criteria: the length of the longest neurite is $>50 \mu \mathrm{m}$ and is two times more than that of the second longest one. Based on these criteria, the percentages of polarized neurons in both Myo X-KD and KIF13B-KD groups were decreased (Fig. 9D,E) and their axons were also shorter (Fig. 9D,F). These results suggest that KIF13B plays a role similar to that of Myo $\mathrm{X}$ in axon initiation.

We next determined whether KIF13B regulates axon projection and branching as Myo X does. To this end, KIF13B was suppressed in E15.5 embryos by IUE of its shRNA (GFP). At neonatal age (e.g., P7 and P14), the neurons electroporated mostly migrated into cortical L2/3 pyramidal neurons whose axons project to the contralateral side via CC (Alcamo et al., 2008). Just as with Myo X-KD axons, axons of KIF13B-KD neurons crossed the midline at $\mathrm{P} 7$, without an obvious reduction in their axonal length (Fig. 9G,H). However, at P14, the axonal contralateral branches were severely diminished in KIF13B-KD neurons compared with those of controls (Fig. 9I,J). Together, these results suggest that KIF13B is necessary to promote axon initiation and branching/targeting in developing cortical neurons, providing additional support for KIF13B as an important mediator for Netrin-1-induced and Myo X-regulated axon initiation and targeting.

\section{Discussion}

In this study, we present evidence that Netrin-1 increases axonal targeting of Myo X in neurons. This event is essential for axon initiation and contralateral branching, but not midline crossing. Further mechanistic studies suggest that Netrin-1 increases Myo $\mathrm{X}$ interaction with KIF13B, thus promoting axonal transport of Myo X, axonal initiation, and branching/targeting. These results reveal a new mechanism underlying Netrin-1-regulated axon pathfinding.

As an unconventional Myosin family protein, Myo $\mathrm{X}$ is widely expressed and implicated in multiple cellular functions in different cell types, including Netrin-1-induced neurite outgrowth and growth cone guidance; Zhu et al., 2007), BMP6-dependent filopodial migration and activation of BMP receptors (Pi et al., 2007), and migration of Xenopus cranial neural crest cells (Hwang et al., 2009; Nie et al., 2009). While it is evident that Myo X modulates growth cone actin dynamics and promotes axon specification in cultured neurons (Yu et al., 2012), the in vivo evidence for this view is limited. Here, we found that Myo X is critical for axon initiation and terminal branching/targeting in the developing neocortex. Myo X KD (by RNA interference) or KO (by Cre-Loxp combination) decreased the axon intensity 
A

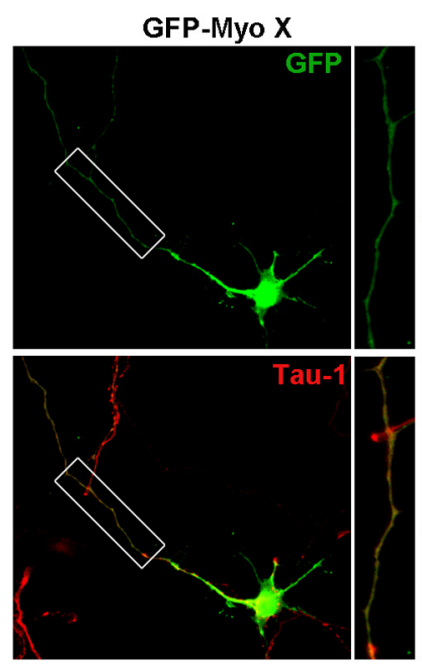

GFP-Myo X/Myc-KIF13B
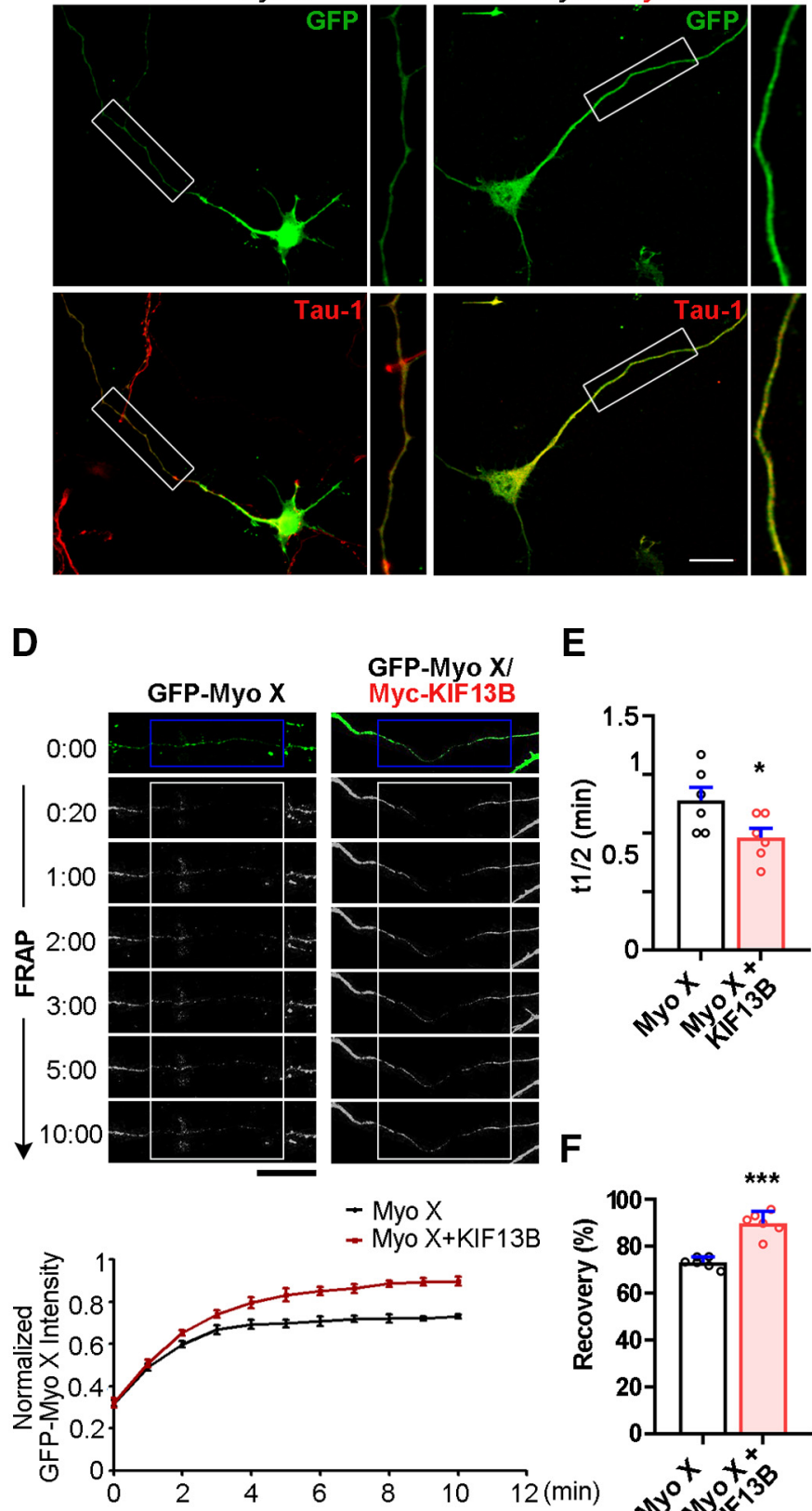

E

B

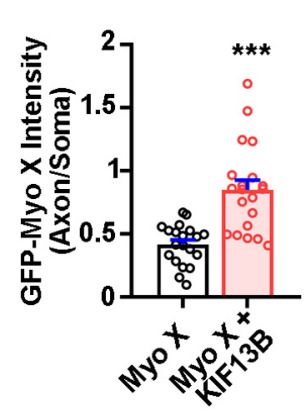

G
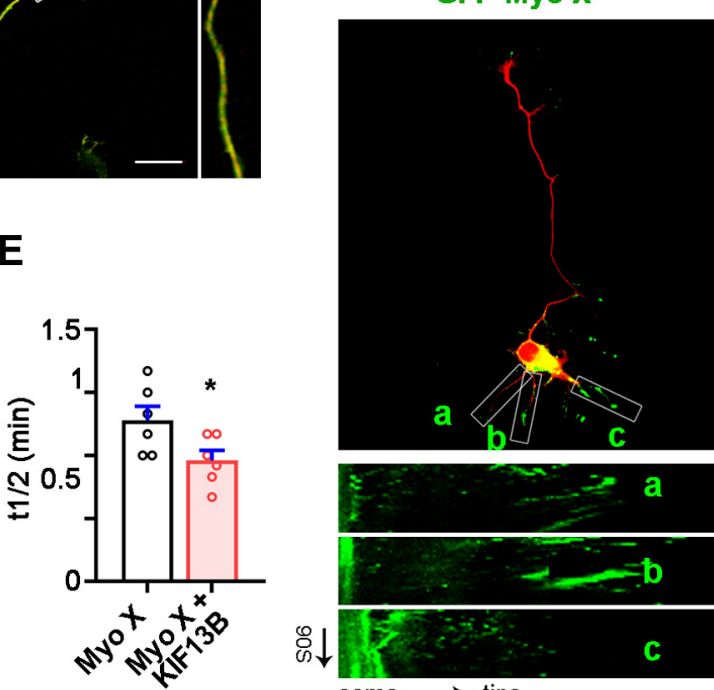

F

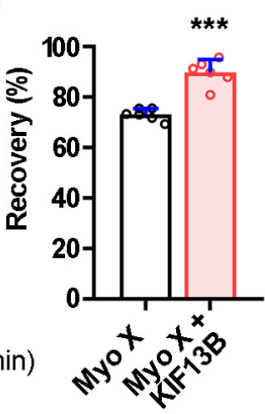

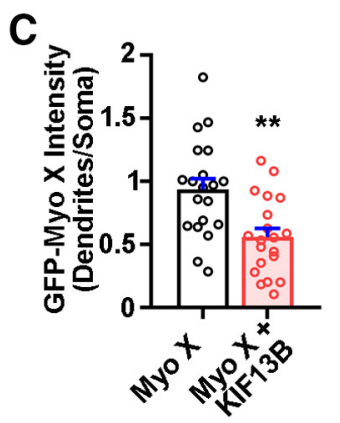

GFP-Myo X/Myc-GAKIN

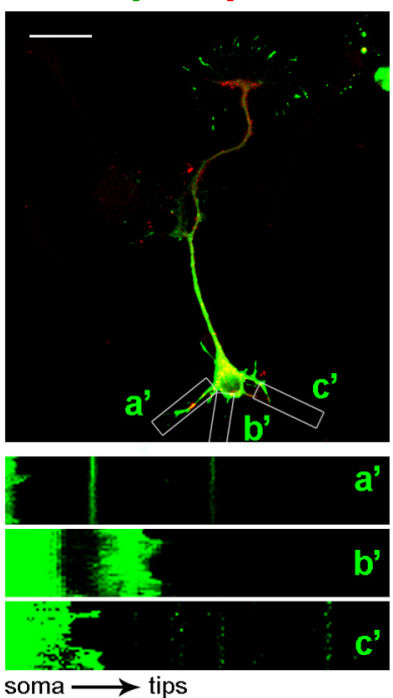

I

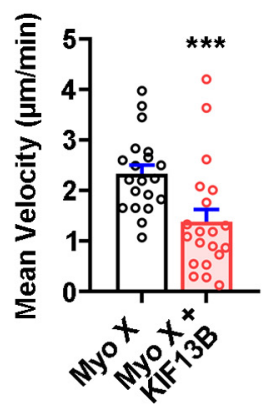

Figure 7. Involvement of KIF13B in Myo X anterograde transportation. $\boldsymbol{A}-\boldsymbol{C}$, Immunostaining analysis using indicated antibodies at DIV3 neurons that were transfected with GFP-Myo X or GFP-Myo X together with Myc-KIF13B at DIV1. A, Images marked in a rectangle were amplified and are shown in the right panels. Scale bar, 20 um. $\boldsymbol{B}$, Quantification of GFP-Myo X intensity in axons. The axonal GFP-Myo X level was normalized to somatic GFP-Myo X. Student's $t$ test, $p<0.0001$. C, Quantification of GFP-Myo X intensity in dendrites. The dendritic GFP-Myo X level was normalized to somatic GFP-Myo X. Student's $t$ test, $p=0.0013$. $\boldsymbol{D}-\boldsymbol{F}$, Analysis of KIF13B effect on axonal GFP-Myo X mobility with FRAP assay. D, Images of FRAP analysis in DIV3 cortical neurons that were transfected with GFP-Myo X or GFP-Myo X together with Myc-KIF13B at DIV1 and the quantification (normalized GFP-Myo X intensity of the photobleached axon compartment). Scale bar, $20 \mu \mathrm{m}$. $\boldsymbol{E}$, Quantification of half-time of maximum recovery. Student's $t$ test, $p=0.0213, n=6$ neurons from three different experiments. $\boldsymbol{F}$, Percentage of GFP-Myo $X$ recovery. Student's $t$ test, $p<0.0001, n=6$ neurons from three different experiments. G-I, Time-lapse imaging analysis of GFP-Myo $X$ together with vector or GFP-Myo $X$ together with Myc-KIF13B-transfected neurons. G, The marked rectangle in $\mathbf{G}$ were further analyzed by kymographs (see bottom panels), which show the mobility of GFP-Myo X-positive vesicles during 5 min recordings. Vertical lines represent stationary Myo X-vesicles; oblique lines or curves to the right represent anterograde movements, and lines to the left indicate retrograde transport. Scale bar, $20 \mu \mathrm{m}$. $\boldsymbol{H}$, Quantification of the mean velocity of GFP-Myo X puncta. Student's $t$ test, $p=0.0026$. I, Quantification of stationary GFP-Myo X. Student's $t$ test, $p=0.0001$. Data are presented as the mean \pm SEM. The numbers of cells scored are from three different brains for each group and are indicated on the graphs. $* p<0.05 ; * * p<0.01 ; * * * p<0.001$.

ratio, suggesting a deficit in axon initiation (Figs. 1E, 9A). Morphology analysis of individual neurons suggested an impairment of axon genesis (Fig. 9D-F). In addition to axon initiation, Myo X KD or KO also impaired axon terminal branching/targeting (Fig. 1C,I). This function of Myo $\mathrm{X}$ is in line with the observations that Myo $\mathrm{X}$ plays an important role in regulating axon filaments and actin filaments in the leading margin of growth cones, which are responsible for perceiving extrinsic guidance factors or adhesive signals and producing traction for axon terminal elongation toward its target (Dent and Gertler, 2003; Yu et al., 2012). Interestingly, Netrin-1 KD in cortical neurons had little effect on the axonal contralateral branching/targeting, while 
A
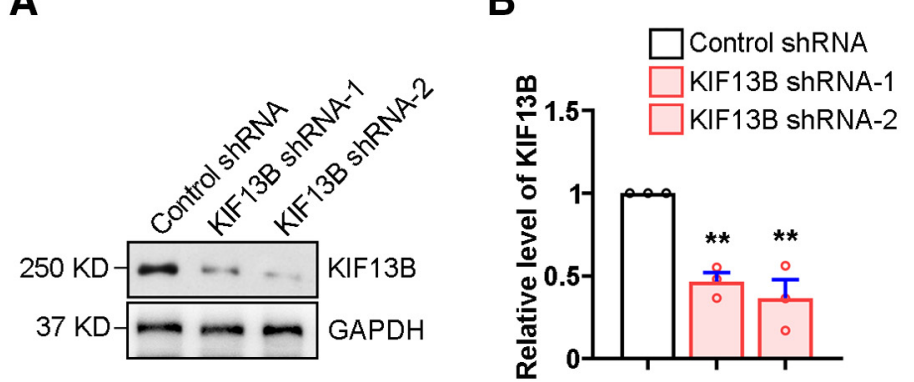

C

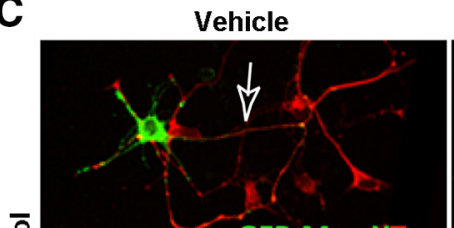

$\overline{\mathrm{o}}$ GFP-Myo XTau
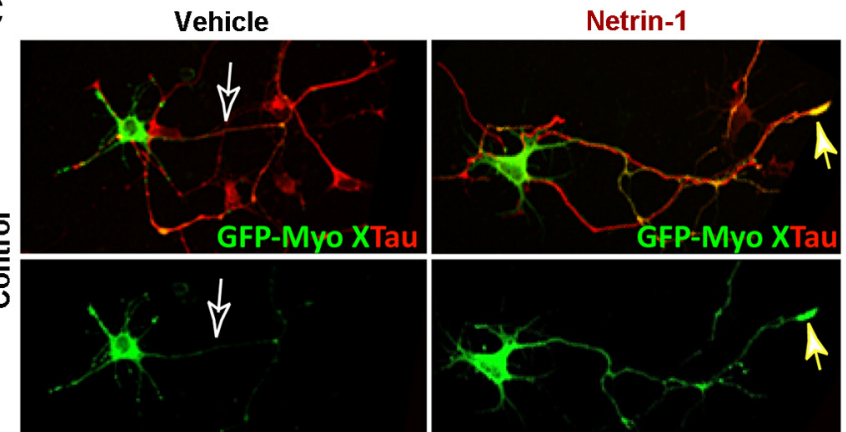

GFP-Myo X
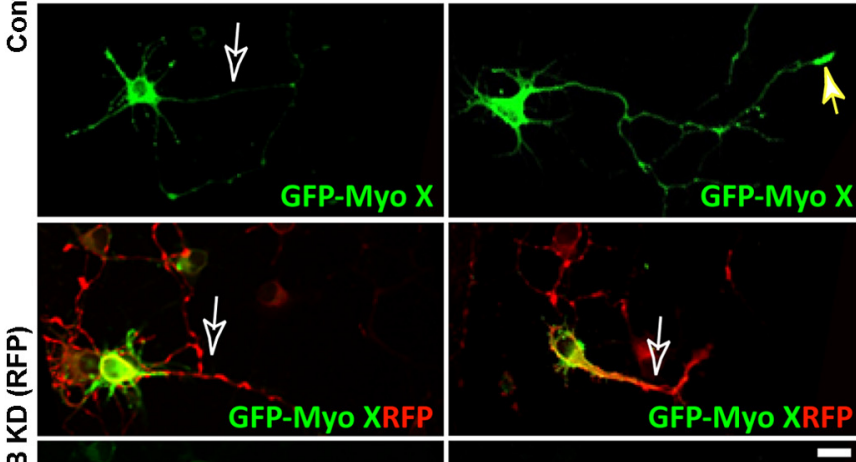

商

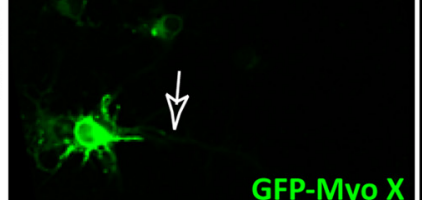

D

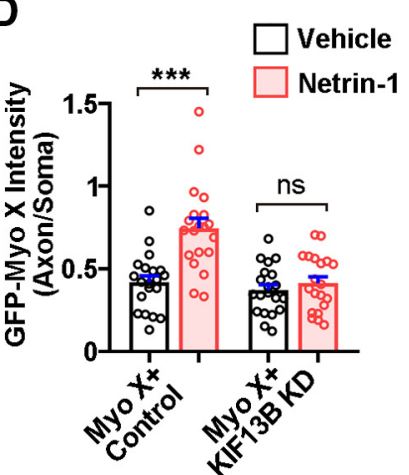

E

Vehicle

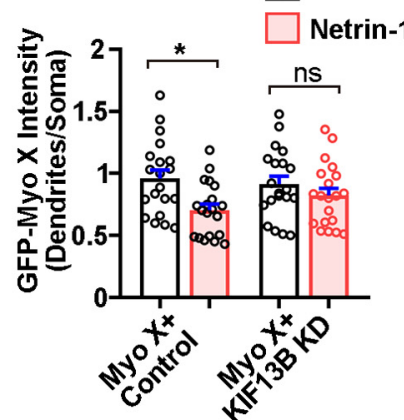

F

E14.5-E17.5
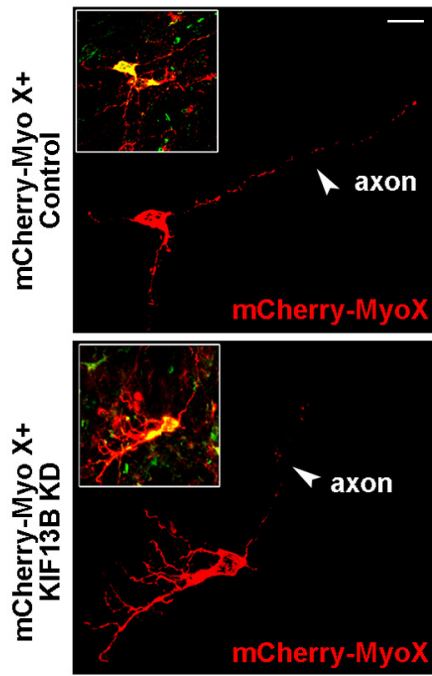

H

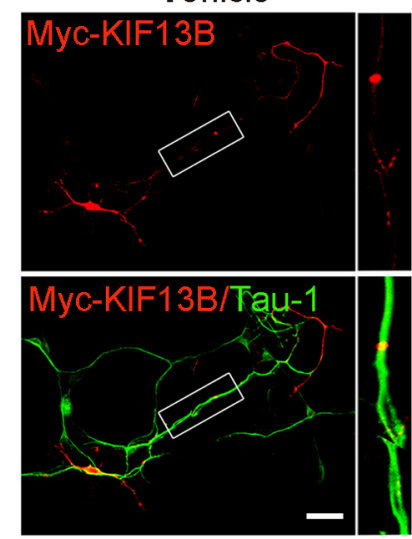

I

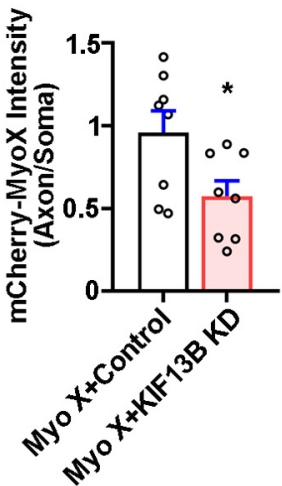

Netrin-1

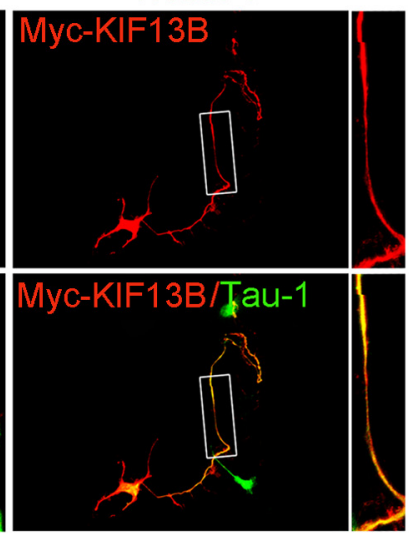

J

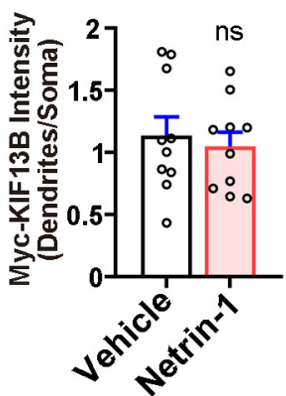

Figure 8. Requirement of KIF13B for Netrin-1 induced axonal distribution of GFP-Myo X. $\boldsymbol{A}$, Western blot showing the silence effect of KIF13B shRNA in cultured cortical neurons. $\boldsymbol{B}$, Quantification of KIF13B knock-down efficiency. One-way ANOVA, $p=0.0036$ for KIF13B shRNA-1 group; $p=0.0015$ for KIF13B shRNA-2 group. C, Neurons transfected with GFP-Myo X together with control shRNA or KIF13B shRNA, respectively, were treated with vehicle or Netrin-1 for $1 \mathrm{~h}$ and subjected to immunostaining at DIV3. Scale bar, $10 \mu \mathrm{m}$. D, Quantification of GFP-Myo X intensity in axons. The axonal GFP-Myo X level was normalized to somatic GFP-Myo X. One-way ANOVA: $p<0.0001$ between GFP-Myo X+Control groups; $p=0.91$ between GFP-Myo $X+$ KIF13B-KD groups. E, Quantification of GFP-Myo X intensity in dendrites. The dendritic GFP-Myo X level was normalized to somatic GFP-Myo X. One-way ANOVA: $p=0.0293$ between GFPMyo $X+$ Control groups; $p=0.88$ between GFP-Myo $X+$ KIF13B-KD groups. $\boldsymbol{F}$, Representative images of cortical neurons electroporated with indicated plasmids at E15.5. Scale bar, $10 \mu$ m. $\mathbf{G}$, Quantification of $\mathrm{mCherry-Myo} X$ intensity in axons. The axonal mCherry-Myo X level was normalized to somatic mCherry-Myo X. Student's $t$ test, $p=0.0331$. $\boldsymbol{H}$, Neurons transfected with MycKIF13B were treated with vehicle or Netrin-1 for $1 \mathrm{~h}$ and subjected to immunostaining with indicated antibodies at DIV3. Images marked in rectangles were amplified and are shown in the right panels. Scale bar, $20 \mu \mathrm{m}$. I, Quantification of Myc-KIF13B intensity in axons. The axonal Myc-KIF13B level was normalized to somatic Myc-KIF13B. Student's $t$ test, $p=0.0026$. $J$, Quantification of Myc-KIF13B intensity in dendrites. The dendritic Myc-KIF13B level was normalized to somatic Myc-KIF13B. Student's $t$ test, $p=0.59$. Data are presented as the mean \pm SEM. The numbers of neurons scored in these groups are from three different experiments and indicated on the graphs. ns, No significant difference; $* p<0.05 ; * * p<0.01 ; * * * p<0.001$. 
A $14.5-\mathrm{E} 18.5$

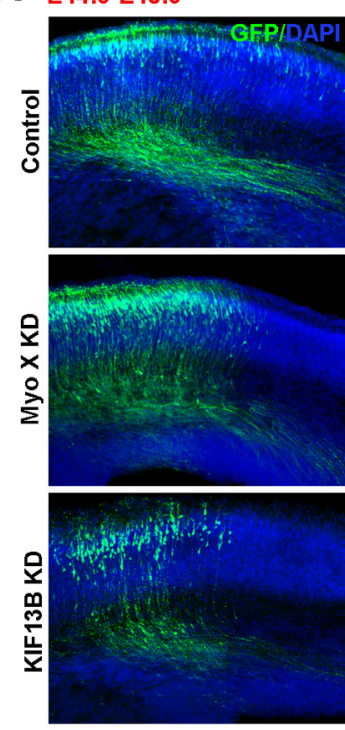

B

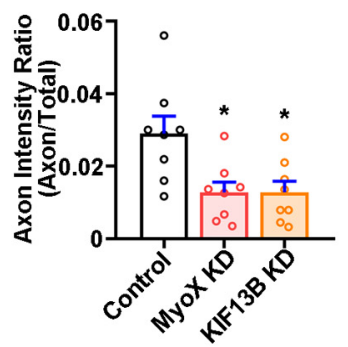

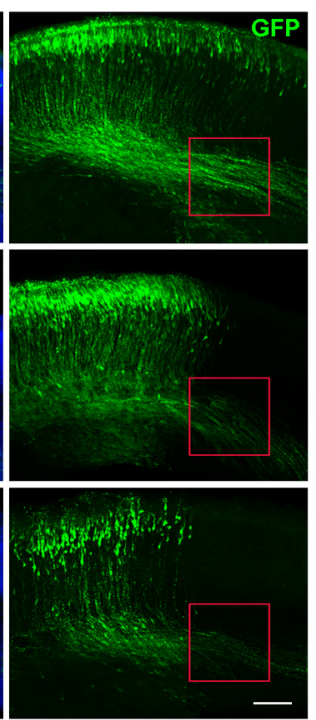

C

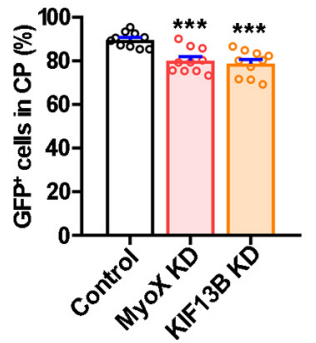

D E15.5-E18.5
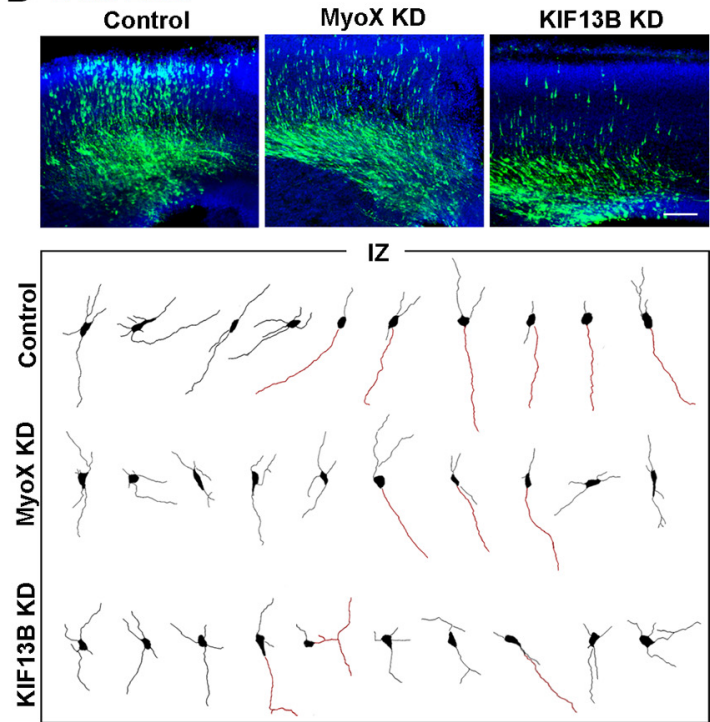

E

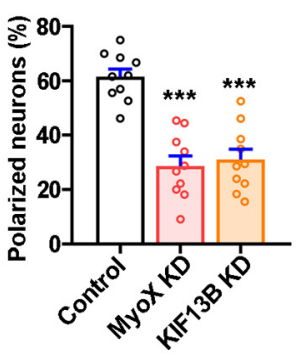

$\mathbf{F}$

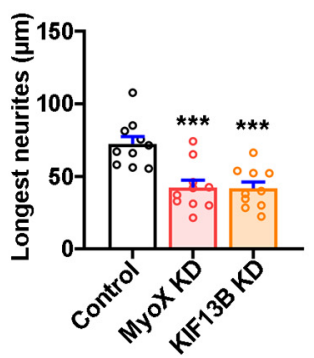

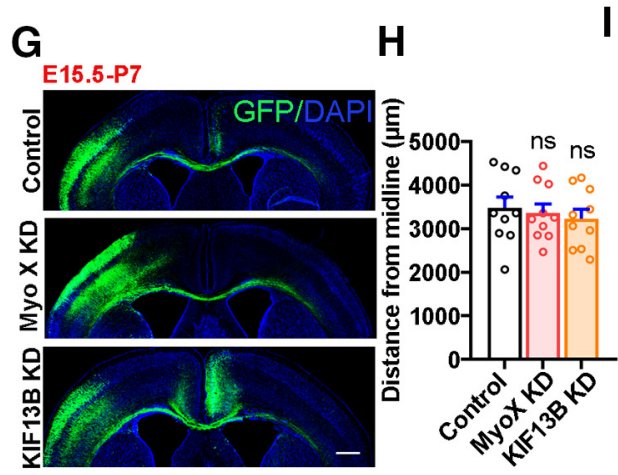

I E15.5-P14

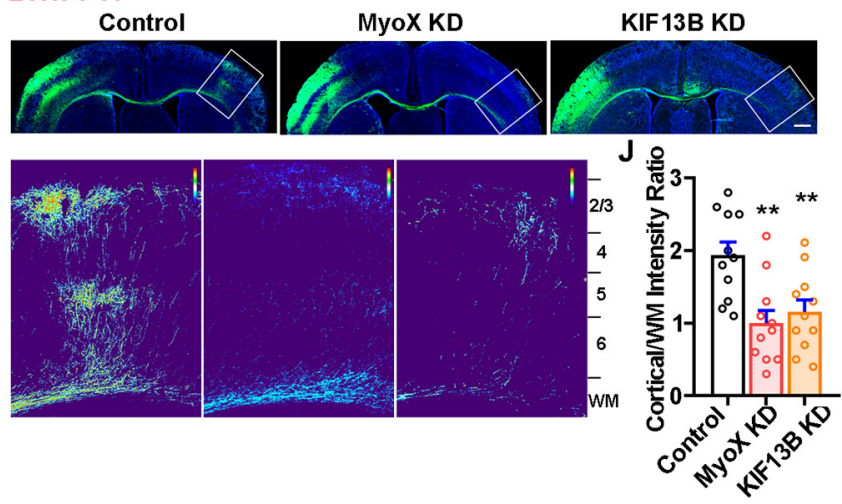

Figure 9. Similar roles of KIF13B in axon initiation and branching as Myo X. A, Representative images of E18.5 cerebral cortex electroporated with control shRNA (Control), KIF13B shRNA (KIF13B-KD), and Myo X miRNA (Myo X-KD) plasmids at E14.5. Scale bar, $100 \mu \mathrm{m}$. B, Quantification of axon initiation by using the axon intensity ratio. Student's $t$ test: for Myo X-KD group, $p=0.0150$; for KIF13B-KD group, $p=0.0102$. C, Quantification of GFP-positive cells in CP. Student's $t$ test: for Myo X-KD group, $p=0.0002$; for KIF13B-KD group, $p<0.0001$. D, Neurons in the IZ of E18.5 cerebral cortex in each group. Mouse cortices electroporated with indicated plasmids were presented in the top panels. The tracings of representative GFP-positive neurons in each group are presented in the bottom panels. The longest neurite (with length $>50 \mu \mathrm{m}$ and two times more than that of the second longest one) is considered as axon and is marked in red. Scale bar, $100 \mu \mathrm{m}$. $\boldsymbol{E}$, Quantification of polarized neurons in IZ. Student's $t$ test: for Myo X-KD group, $p<0.0001$; for KIF13B-KD group, $p<0.0001$. $\boldsymbol{F}$, Quantification of the length of longest neurites. Student's $t$ test: for Myo X-KD group, $p=0.0003$; for KIF13B-KD group, $p=0.0003$. $\mathbf{G}, \boldsymbol{H}$, Representative images of P7 cerebral cortex electroporated with above indicated plasmids at E15.5 as well as quantification of axon elongation. Scale bar, $500 \mu \mathrm{m}$. Student's $t$ test: for Myo X-KD group, $p=0.9059$; for KIF13B-KD group, $p=0.65$. I, J, Representative images of P14 cerebral cortex electroporated with above indicated plasmids at E15.5 as well as quantification of axon contralateral branching. Scale bar, $100 \mu \mathrm{m}$. Student's $t$ test: for Myo X-KD group, $p=0.0013$; for KIF13B-KD group, $p=0.0067$. Data are presented as the mean \pm SEM. The numbers of brain sections or cells scored are from three different brains for each group and indicated on the graphs. ns, No significant difference; $* p<0.05 ; * * p<0.01 ; * * * p<0.001$.

DCC KD impaired axonal contralateral branching/targeting (Fig. $2 F, G)$. These results together suggest that DCC and Myo X in neurons play a cell-autonomous role in promoting axon branching, while Netrin-1, as an extracellular cue, regulates axon development in a nonautonomous way. Although Netrin-1DCC-Myo X was involved in axon branching, it is not the only pathway underlying axon branching. It is of interest to note that Netrin-1 promotes exocytosis and plasma membrane expansion 


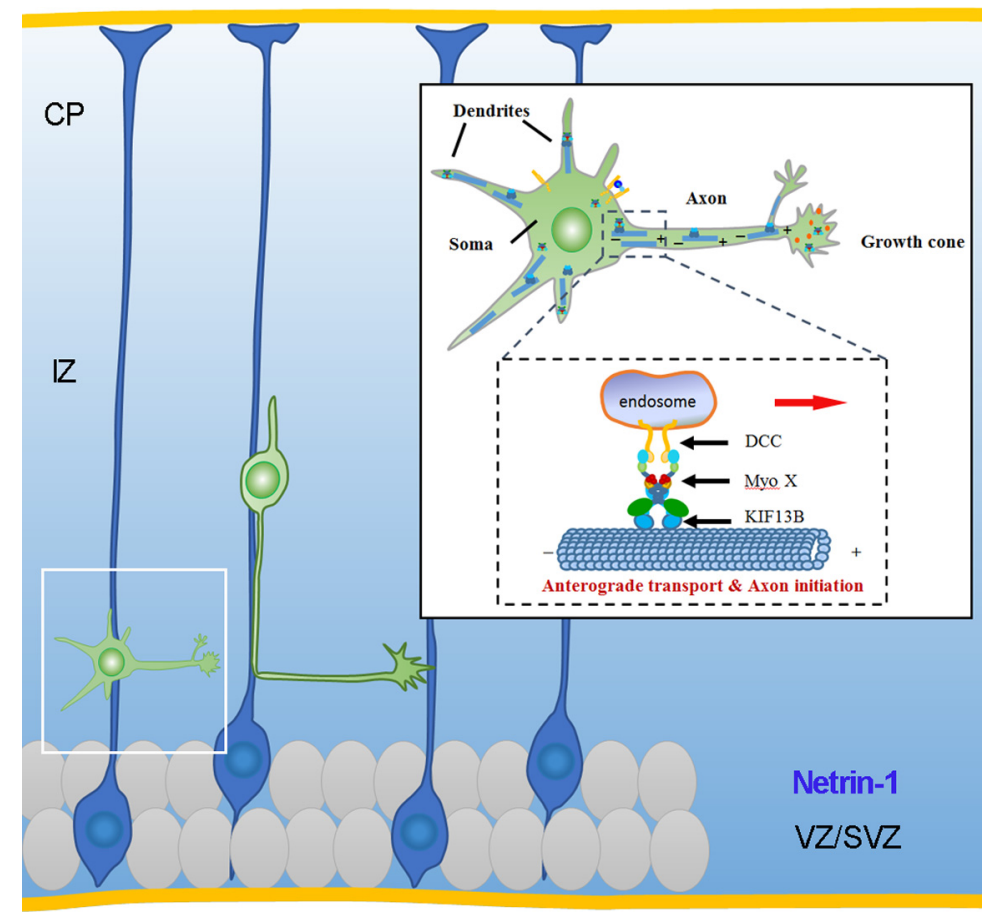

Figure 10. Graphical abstract.

for axon branching via TRIM9 release of SNAP25 and SNAREmediated vesicle fusion (Winkle et al., 2014). It will be of interest to investigate whether Myo X is involved in Netrin-1-stimulated exocytosis for axon branching. Notice that Myo X KO or $\mathrm{KD}$ has little effect on axon midline crossing, as it does in Netrin-1-KO or DCC-KD axons (Fig. $2 F, G$ ). These results suggest a neuronal DCC-Myo X-independent mechanism for axon midline crossing.

Axons contain abundant microtubules, although their growth cones have enriched actin filaments (Dent and Gertler, 2003). How is Myo X, an actin filament-based motor protein, transported to the growth cones of axons? Although Myo X interacts with microtubules with its MyTH4 domain (Weber et al., 2004; Woolner et al., 2008; Wühr et al., 2008), little evidence demonstrates that Myo X has microtubule-based motor activity. Thus, we speculate that the microtubule-dependent motor protein kinesin may be responsible for Myo X anterograde transportation in axons. To this end, KIF13B was identified as a Myo X binding partner to be responsible for Myo X anterograde transportation. Interestingly, KIF13B, a kinesin family motor protein, plays an essential role in anterograde transport of $\mathrm{PI}(3,4,5) \mathrm{P} 3$ (Horiguchi et al., 2006), a binding partner and regulator of Myo X (Figs. 6, 7). Moreover, KIF13B exerts functions similar to those of Myo X in promoting axon initiation and terminal targeting (Fig. 9). In aggregates, our results suggest that Myo X appears to be a cargo of KIF13B during its axonal transportation, and at the same time, the actin-based motor activity of Myo $\mathrm{X}$ is suppressed by KIF13B.

Netrin-1/DCC signaling is involved in many aspects of axon development, including axon outgrowth and guidance, growth cone steering, and axon branching. The canonical model for the function of Netrin- 1 in axon guidance is that Netrin- 1 acts as a long-range diffusible guidance cue, centered in the midline (e.g., floor plate in the developing spinal cord), attracting or repulsing axons for their midline crossing. Recent studies have shown that Netrin- 1 is produced not only in the midline, but also in neural progenitor cells in the VZ, and is deposited on the pial surface as a haptotactic adhesive substrate, where it guides DCCpositive axon growth (Dominici et al., 2017). In developing cerebral cortex, Netrin-1 mRNA is highly expressed in VZ/SVZ (Zhang et al., 2018). It is of interest to note the report by Brignani et al. (2020) that Netrin-1, produced in the forebrain and provided to the midbrain through axon projections, instructs the migration of GABAergic neurons into the ventral $\mathrm{SN}$, which supports the expression of Netrin-1 in developing neurons in forebrain (Brignani et al., 2020). In line with these reports are observations by Allen's in situ and single-cell RNA-seq databases on the websites. These results implicate that Netrin-1/DCC signaling in local microenvironment surrounding newborn neurons is important for axon development. In line with this view, we found that the axon initiation was slowed down by Netrin-1 KO in the local region (Fig. $2 A, B)$, and Netrin-1 overexpression diminished Myo X deficiency-induced axon initiation deficit. Netrin-1 was knocked out in neural stem cells, as well as their progeny, including neurons and glial cells, which likely reduce Netrin- 1 in the local environment. Therefore, the defect in Netrin-1-KO neurons could be because of loss of the autocrine and/or local paracrine effect of Netrin-1. How does Netrin-1 rescue the axon initiation deficit in Myo X-deficient neurons? It is noteworthy that neurons with Myo X KD by its miRNA, unlike Myo X KO, still contained low levels of Myo X. Upon Netrin-1 overexpression, it may promote the axonal transportation of the remaining Myo X and thus diminish the axon initiation defect. Alternatively, Netrin-1 may increase axonal initiation in a Myo $\mathrm{X}$-independent manner, as multiple downstream signals can be activated by Netrin- 1 .

In light of our results, we speculate the existence of the DCCMyo X-KIF13B complex. Netrin-1 may increase the complex formation by generating more $\mathrm{PI}(3,4,5) \mathrm{P} 3$, which binds to Myo $\mathrm{X}$, changes Myo X conformation for DCC and KIF13B binding, and then undergoes anterograde transport along microtubules (Fig. 10). The motor activity of Myo X may be suppressed by disconnecting Myo X with F-actin filaments, as we can see that KIF13B suppress Myo X motility in dendrite-like actin filaments. In this complex, Myo X acts as a central adapter protein to link its cargos of DCC and PI(3,4,5)P3/PI(3,4)P2 with KIF13B. Such a Myo X-containing complex may be crucial for Netrin-1induced axonal outgrowth and growth cone-attractive response.

\section{References}

Ackerman SL, Kozak LP, Przyborski SA, Rund LA, Boyer BB, Knowles BB (1997) The mouse rostral cerebellar malformation gene encodes an UNC-5-like protein. Nature 386:838-842.

Alcamo EA, Chirivella L, Dautzenberg M, Dobreva G, Fariñas I, Grosschedl R, McConnell SK (2008) Satb2 regulates callosal projection neuron identity in the developing cerebral cortex. Neuron 57:364-377.

Barnes AP, Polleux F (2009) Establishment of axon-dendrite polarity in developing neurons. Annu Rev Neurosci 32:347-381.

Berg JS, Cheney RE (2002) Myosin-X is an unconventional myosin that undergoes intrafilopodial motility. Nat Cell Biol 4:246-250. 
Berg JS, Derfler BH, Pennisi CM, DP C, Cheney RE (2000) Myosin-X, a novel myosin with pleckstrin homology domains, associates with regions of dynamic actin. J Cell Sci 19:3439-3451.

Braisted JE, Catalano SM, Stimac R, Kennedy TE, Tessier-Lavigne M, Shatz CJ, O'Leary DD (2000) Netrin-1 promotes thalamic axon growth and is required for proper development of the thalamocortical projection. J Neurosci 20:5792-5801.

Brignani S, Raj DDA, Schmidt ERE, Düdükcü Ö, Adolfs Y, De Ruiter AA, Rybiczka-Tesulov M, Verhagen MG, van der Meer C, Broekhoven MH, Moreno-Bravo JA, Grossouw LM, Dumontier E, Cloutier J-F, Chédotal A, Pasterkamp RJ (2020) Remotely produced and axon-derived Netrin-1 instructs GABAergic neuron migration and dopaminergic substantia nigra development. Neuron 107:684-702.

Campbell DS, Holt CE (2003) Apoptotic pathway and MAPKs differentially regulate chemotropic responses of retinal growth cones. Neuron 37:939952.

Chen J-Y, He X-X, Ma C, Wu X-M, Wan X-L, Xing Z-K, Pei Q-Q, Dong XP, Liu D-X, Xiong W-C, Zhu X-J (2017) Netrin-1 promotes glioma growth by activating NF- $\kappa$ B via UNC5A. Sci Rep 7:5454.

Colamarino SA, Tessier-Lavigne M (1995) The role of the floor plate in axon guidance. Annu Rev Neurosci 18:497-529.

Cox D, Berg JS, Cammer M, Chinegwundoh JO, Dale BM, Cheney RE, Greenberg S (2002) Myosin X is a downstream effector of PI(3)K during phagocytosis. Nat Cell Biol 4:469-477.

Culotti JG, Merz DC (1998) DCC and netrins. Curr Opin Cell Biol 10:609613.

Dent EW, Gertler FB (2003) Cytoskeletal dynamics and transport in growth cone motility and axon guidance. Neuron 40:209-227.

Dominici C, Moreno-Bravo JA, Puiggros SR, Rappeneau Q, Rama N, Vieugue P, Bernet A, Mehlen P, Chédotal A (2017) Floor-plate-derived netrin-1 is dispensable for commissural axon guidance. Nature 545:350-354.

Dotti CG, Sullivan CA, Banker GA (1988) The establishment of polarity by hippocampal neurons in culture. J Neurosci 8:1454-1468.

Fenlon LR, Richards LJ (2015) Contralateral targeting of the corpus callosum in normal and pathological brain function. Trends Neurosci 38:264-272.

Forcet C, Stein E, Pays L, Corset V, Llambi F, Tessier-Lavigne M, Mehlen P (2002) Netrin-1-mediated axon outgrowth requires deleted in colorectal cancer-dependent MAPK activation. Nature 417:443-447.

Hedgecock EM, Culotti JG, Hall DH (1990) The unc-5, unc-6, and unc-40 genes guide circumferential migrations of pioneer axons and mesodermal cells on the epidermis in C. elegans. Neuron 4:61-85.

Hirokawa N, Niwa S, Tanaka Y (2010) Molecular motors in neurons: transport mechanisms and roles in brain function, development, and disease. Neuron 68:610-638.

Hong K, Hinck L, Nishiyama M, Poo MM, Tessier-Lavigne M, Stein E (1999) A ligand-gated association between cytoplasmic domains of UNC5 and DCC family receptors converts netrin-induced growth cone attraction to repulsion. Cell 97:927-941.

Horiguchi K, Hanada T, Fukui Y, Chishti AH (2006) Transport of PIP3 by GAKIN, a kinesin-3 family protein, regulates neuronal cell polarity. J Cell Biol 174:425-436.

Hwang YS, Luo T, Xu Y, Sargent TD (2009) Myosin-X is required for cranial neural crest cell migration in Xenopus laevis. Dev Dyn 238:2522-2529.

Keleman K, Dickson BJ (2001) Short- and long-range repulsion by the Drosophila Unc5 netrin receptor. Neuron 32:605-617.

Kerber ML, Cheney RE (2011) Myosin-X: a MyTH-FERM myosin at the tips of filopodia. J Cell Sci 124:3733-3741.

Kwan KY, Sestan N, Anton ES (2012) Transcriptional co-regulation of neuronal migration and laminar identity in the neocortex. Development 139:1535-1546.

Lai M, Guo Y, Ma J, Yu H, Zhao D, Fan W, Ju X, Sheikh MA, Malik YS, Xiong W, Guo W, Zhu X (2015) Myosin X regulates neuronal radial migration through interacting with N-cadherin. Front Cell Neurosci 9:326.

Leonardo ED, Hinck L, Masu M, Keino-Masu K, Ackerman SL, TessierLavigne M (1997) Vertebrate homologues of C. elegans UNC-5 are candidate netrin receptors. Nature 386:833-838.

Li W, Lee J, Vikis HG, Lee S-H, Liu G, Aurandt J, Shen T-L, Fearon ER, Guan J-L, Han M, Rao Y, Hong K, Guan K-L (2004) Activation of FAK and $\mathrm{Src}$ are receptor-proximal events required for netrin signaling. Nat Neurosci 7:1213-1221.

Li X, Saint-Cyr-Proulx E, Aktories K, Lamarche-Vane N (2002) Rac1 and Cdc42 but not RhoA or Rho kinase activities are required for neurite outgrowth induced by the Netrin-1 receptor DCC (deleted in colorectal cancer) in N1E-115 neuroblastoma cells. J Biol Chem 277:15207-15214.

Liu G, Beggs H, Jürgensen C, Park HT, Tang H, Gorski J, Jones KR, Reichardt LF, Wu J, Rao Y (2004) Netrin requires focal adhesion kinase and Src family kinases for axon outgrowth and attraction. Nat Neurosci 7:1222-1232.

Liu Y, Peng Y, Dai PG, Du QS, Mei L, Xiong WC (2012) Differential regulation of myosin X movements by its cargos, DCC and neogenin. J Cell Sci 125:751-762.

Ma J, Zhang LQ, He ZX, He XX, Wang YJ, Jian YL, Wang X, Zhang BB, Su C, Lu J, Huang BQ, Zhang Y, Wang GY, Guo WX, Qiu DL, Mei L, Xiong WC, Zheng YW, Zhu XJ (2019) Autism candidate gene DIP2A regulates spine morphogenesis via acetylation of cortactin. PLoS Biol 17:e3000461.

Ming G, Song H, Berninger B, Inagaki N, Tessier-Lavigne M, Poo M (1999) Phospholipase C-gamma and phosphoinositide 3-kinase mediate cytoplasmic signaling in nerve growth cone guidance. Neuron 23:139-148.

Ming GL, Wong ST, Henley J, Yuan XB, Song HJ, Spitzer NC, Poo MM (2002) Adaptation in the chemotactic guidance of nerve growth cones. Nature 417:411-418.

Namba T, Kibe Y, Funahashi Y, Nakamuta S, Takano T, Ueno T, Shimada A, Kozawa S, Okamoto M, Shimoda Y, Oda K, Wada Y, Masuda T, Sakakibara A, Igarashi M, Miyata T, Faivre-Sarrailh C, Takeuchi K, Kaibuchi K (2014) Pioneering axons regulate neuronal polarization in the developing cerebral cortex. Neuron 81:814-829.

Namba T, Funahashi Y, Nakamuta S, Xu C, Takano T, Kaibuchi K (2015) Extracellular and intracellular signaling for neuronal polarity. Physiol Rev 95:995-1024.

Nie S, Kee Y, Bronner-Fraser M (2009) Myosin-X is critical for migratory ability of Xenopus cranial neural crest cells. Dev Biol 335:132-142.

Pi X, Ren R, Kelley R, Zhang C, Moser M, Bohil AB, Divito M, Cheney RE, Patterson C (2007) Sequential roles for myosin-X in BMP6-dependent filopodial extension, migration, and activation of BMP receptors. J Cell Biol 179:1569-1582.

Plantard L, Arjonen A, Lock JG, Nurani G, Ivaska J, Strömblad S (2010) $\operatorname{Ptd} \operatorname{Ins}(3,4,5) \mathrm{P}(3)$ is a regulator of myosin-X localization and filopodia formation. J Cell Sci 123:3525-3534.

Ren XR, Du QS, Huang YZ, Ao SZ, Mei L, Xiong WC (2001) Regulation of CDC42 GTPase by proline-rich tyrosine kinase 2 interacting with PSGAP, a novel pleckstrin homology and Src homology 3 domain containing rhoGAP protein. J Cell Biol 152:971-984.

Ren X-R, Ming G-L, Xie Y, Hong Y, Sun D-M, Zhao Z-Q, Feng Z, Wang Q, Shim S, Chen Z-F, Song H-J, Mei L, Xiong W-C (2004) Focal adhesion kinase in netrin-1 signaling. Nat Neurosci 7:1204-1212.

Shekarabi M, Kennedy TE (2002) The netrin-1 receptor DCC promotes filopodia formation and cell spreading by activating Cdc42 and Rac1. Mol Cell Neurosci 19:1-17.

Tagawa Y, Hirano T (2012) Activity-dependent callosal axon projections in neonatal mouse cerebral cortex. Neural Plast 2012:797295.

Tokuo H, Ikebe M (2004) Myosin X transports Mena/VASP to the tip of filopodia. Biochem Biophys Res Commun 319:214-220.

Tokuo H, Mabuchi K, Ikebe M (2007) The motor activity of myosin-X promotes actin fiber convergence at the cell periphery to initiate filopodia formation. J Cell Biol 179:229-238.

Umeki N, Jung HS, Sakai T, Sato O, Ikebe R, Ikebe M (2011) Phospholipiddependent regulation of the motor activity of myosin X. Nat Struct Mol Biol 18:783-788.

Wang B, Pan JX, Yu H, Xiong L, Zhao K, Xiong S, Guo JP, Lin S, Sun D, Zhao L, Guo H, Mei L, Xiong WC (2019) Lack of myosin X enhances osteoclastogenesis and increases cell surface Unc5b in osteoclast-lineage cells. J Bone Miner Res 34:939-954.

Weber KL, Sokac AM, Berg JS, Cheney RE, Bement WM (2004) A microtubule-binding myosin required for nuclear anchoring and spindle assembly. Nature 431:325-329.

Winkle CC, McClain LM, Valtschanoff JG, Park CS, Maglione C, Gupton SL (2014) A novel Netrin-1-sensitive mechanism promotes local SNAREmediated exocytosis during axon branching. J Cell Biol 205:217-232.

Woolner S, O'Brien LL, Wiese C, Bement WM (2008) Myosin-10 and actin filaments are essential for mitotic spindle function. J Cell Biol 182:77-88.

Wühr M, Mitchison TJ, Field CM (2008) Mitosis: new roles for myosin-X and actin at the spindle. Curr Biol 18:R912-914.

Xie Y, Ding Y-Q, Hong Y, Feng Z, Navarre S, Xi C-X, Zhu X-J, Wang C-L, Ackerman SL, Kozlowski D, Mei L, Xiong W-C (2005) Phos- 
phatidylinositol transfer protein-alpha in netrin-1-induced PLC signalling and neurite outgrowth. Nat Cell Biol 7:1124-1132.

Xie Y, Hong Y, Ma XY, Ren XR, Ackerman S, Mei L, Xiong WC (2006) DCC-dependent phospholipase $\mathrm{C}$ signaling in netrin-1-induced neurite elongation. J Biol Chem 281:2605-2611.

Yogev S, Shen K (2017) Establishing neuronal polarity with environmental and intrinsic mechanisms. Neuron 96:638-650.

Yonezawa S, Kimura A, Koshiba S, Masaki S, Ono T, Hanai A, Sonta S, Kageyama T, Takahashi T, Moriyama A (2000) Mouse myosin X: molecular architecture and tissue expression as revealed by northern blot and in situ hybridization analyses. Biochem Biophys Res Commun 271:526-533.

Yoshimura Y, Terabayashi T, Miki H (2010) Par1b/MARK2 phosphorylates kinesin-like motor protein GAKIN/KIF13B to regulate axon formation. Mol Cell Biol 30:2206-2219.
Yu H, Wang N, Ju X, Yang Y, Sun D, Lai M, Cui L, Sheikh MA, Zhang J, Wang X, Zhu X (2012) PtdIns (3,4,5) P3 recruitment of Myo10 is essential for axon development. PLoS One 7:e36988.

Zhang H, Berg JS, Li Z, Wang Y, Lång P, Sousa AD, Bhaskar A, Cheney RE, Strömblad S (2004) Myosin-X provides a motor-based link between integrins and the cytoskeleton. Nat Cell Biol 6:523-531.

Zhang J-H, Zhao Y-F, He X-X, Zhao Y, He Z-X, Zhang L, Huang Y, Wang Y-B, Hu L, Liu L, Yu H-L, Xu J-H, Lai M-M, Zhao D-D, Cui L, Guo WX, Xiong W-C, Ding Y-Q, Zhu X-J (2018) DCC-mediated Dabl phosphorylation participates in the multipolar-to-bipolar transition of migrating neurons. Cell Rep 22:3598-3611.

Zhu XJ, Wang CZ, Dai PG, Xie Y, Song NN, Liu Y, Du QS, Mei L, Ding YQ, Xiong WC (2007) Myosin X regulates netrin receptors and functions in axonal path-finding. Nat Cell Biol 9:184-192. 